\title{
Genome-wide analysis of BpDof genes and the tolerance to drought stress in birch (Betula platyphylla)
}

\author{
Shilin Sun ${ }^{\text {Equal first author, } 1}$, Bo Wang ${ }^{\text {Equal first author, } 2}{ }^{2}$, Qi Jiang ${ }^{2}$, Zhuoran Li ${ }^{1}$, Site Jia ${ }^{1}$, Yucheng Wang ${ }^{1}$, Huiyan Guo ${ }^{\text {Corresp. } 1}$ \\ ${ }^{1}$ The Key Laboratory of forest tree genetics, breeding and Cultivation of Liaoning Province, Shenyang Agricultural University, Shenyang, Liaoning, China \\ 2 Department of Life Science and Technology, Mudanjiang Normal University, Mudanjiang, Heilongjiang, China \\ Corresponding Author: Huiyan Guo \\ Email address: Ixyghy@syau.edu.cn
}

Background. DNA binding with one finger (Dof) proteins are plant-specific transcription factors playing vital roles in developmental processes and stress responses in plants. Nevertheless, the characterizations, expression patterns, and functions of the Dof family under drought stress (a key determinant of plant physiology and metabolic homeostasis) in woody plants remain unclear.

Methods. The birch (Betula platyphylla var. mandshuric) genome and plant TFDB database were used to identify Dof gene family members in birch plants. ClustalW2 of BioEdit v7.2.1, MEGA v7.0, ExPASy ProtParam tool, Subloc, TMHMM v2.0, GSDS v2.0, MEME, TBtools, KaKs Calculator v2.0, and PlantCARE were respectively used to align the $B p D$ of sequences, build a phylogenetic tree, identify the physicochemical properties, analyze the chromosomal distribution and synteny, and identify the ciselements in the promoter regions of the 26 BpDof genes. Besides, the birch seedlings were exposed to PEG6000-simulated drought stress, and the expression patterns of the BpDof genes in different tissues were analyzed by qRT-PCR. The histochemical staining and the evaluation of physiological indexes were performed to assess the plant tolerance to drought with transient overexpression of BpDof4, BpDof11, and BpDof17 genes. SPSS software and ANOVA were used to conduct all statistical analyses and determine statistically significant differences between results.

Results. A total of 26 BpDof genes were identified in birch via whole-genome analysis. The conserved Dof domain with a $C(x) 2 C(x) 21 C(x) 2 C$ zinc finger motif was present in all BpDof proteins. These birch $B p D o f s$ were classified into four groups (A to D) according to the phylogenetic analysis of Arabidopsis thaliana Dof genes. BpDof proteins within the same group mostly possessed similar motifs, as detected by conserved motif analysis. The exon-intron analysis revealed that the structures of $B p D$ of genes differed, indicating probable gene gain and lose during the BpDof evolution. The chromosomal distribution and synteny analysis showed that the 26 BpDofs were unevenly distributed on 14 chromosomes, and seven duplication events among six chromosomes were found. Cis-acting elements were abundant in the promoter regions of the 26 BpDof genes. qRT-PCR revealed that the expression of the 26 BpDof genes was differentially regulated by drought stress among roots, stems, and leaves. Most $B p D$ of genes responded to drought stress, and $B p D$ of $4, B p D o f 11$, and $B p D o f 17$ were significantly upregulated. Therefore, plants overexpressing these three genes were generated to investigate drought stress tolerance. The BpDof4-, BpDof11-, and BpDof17-overexpressing plants showed promoted reactive oxygen species (ROS) scavenging capabilities and less severe cell damage, suggesting that they conferred enhanced drought tolerance in birch. This study provided an in-depth insight into the structure, evolution, expression, and function of the Dof gene family in plants. 


\section{Genome-wide analysis of $B p D o f$ genes and the tolerance to 2 drought stress in birch (Betula platyphylla)}

3 Keywords: Drought stress $\cdot$ Betula platyphylla $\cdot$ Dof transcription factor $\cdot$ Expression analysis

4 Key Message: The genome-wide characterization of $26 \mathrm{BpDof}$ genes, expression patterns in

5 different tissues, and tolerance to drought stress in birch provided novel information about the

6 structure, evolution, expression, and function of the Dof gene family in plants. 


\section{Abstract}

8 Background. DNA binding with one finger (Dof) proteins are plant-specific transcription

9 factors playing vital roles in developmental processes and stress responses in plants.

10 Nevertheless, the characterizations, expression patterns, and functions of the Dof family

11 under drought stress (a key determinant of plant physiology and metabolic homeostasis) in

12 woody plants remain unclear.

13 Methods. The birch (Betula platyphylla) genome and plant TFDB database were used to

14 identify Dof gene family members in birch plants. ClustalW2 of BioEdit v7.2.1, MEGA v7.0,

15 ExPASy ProtParam tool, Subloc, TMHMM v2.0, GSDS v2.0, MEME, TBtools, KaKs

16 Calculator v2.0, and PlantCARE were respectively used to align the BpDof sequences, build a phylogenetic tree, identify the physicochemical properties of the BpDof proteins, analyze the chromosomal distribution and synteny of $B p D o f$ s, and identify the cis-elements in the promoter regions of the 26 BpDof genes. Besides, the birch seedlings were exposed to PEG6000-simulated drought stress, and the expression patterns of the 26 BpDof genes in different tissues were analyzed by qRT-PCR. The histochemical staining and the evaluation of physiological indexes were performed to assess the plant tolerance to drought with transient overexpression of BpDof4, BpDof11, and BpDof17 genes. SPSS software and ANOVA were used to conduct all statistical analyses and determine statistically significant differences between results.

Results. A total of 26 BpDof genes were identified in birch (B. platyphylla var. mandshuric) via whole-genome analysis. The conserved Dof domain with a $\mathrm{C}(\mathrm{x}) 2 \mathrm{C}(\mathrm{x}) 21 \mathrm{C}(\mathrm{x}) 2 \mathrm{C}$ zinc finger motif was present in all BpDof proteins. These birch BpDofs were classified into four groups (A to D) according to the phylogenetic analysis of Arabidopsis thaliana Dof genes. BpDof proteins within the same group mostly possessed similar motifs, as detected by conserved motif analysis. The exon-intron analysis revealed that the structures of $B p D o f$ genes differed, indicating probable gene gain and lose during the BpDof evolution. The 
chromosomal distribution and synteny analysis showed that the $26 B p D o f$ s were unevenly distributed on 14 chromosomes, and seven duplication events among six chromosomes were found. Cis-acting elements were abundant in the promoter regions of the 26 BpDof genes, such as MBS, MYB, G-Box, GT1-motif, ABRE, and ERE. qRT-PCR revealed that the expression of the 26 BpDof genes was differentially regulated by drought stress among roots, stems, and leaves. Most BpDof genes responded to drought stress, and BpDof4, BpDof11, and BpDof17 were significantly up-regulated. Therefore, plants overexpressing these three genes were generated to investigate drought stress tolerance. The BpDof4-, BpDof11-, and BpDof17-overexpressing plants showed promoted reactive oxygen species (ROS) scavenging capabilities and less severe cell damage, suggesting that they conferred enhanced drought tolerance in birch. This study provided an in-depth insight into the structure, evolution, expression, and function of the Dof gene family in plants.

\section{Introduction}

Transcriptional regulation of gene expression in plants has a vital role in controlling or influencing many critical biological processes, such as cellular morphogenesis, signal transduction, and adverse environmental stress responses (Borrego-Benjumea et al. 2020; Riechmann \& Ratcliffe 2000; Yang et al. 2020). Transcription factors (TFs) can control the expression of genes by binding to specific cis-elements in their promoter regions and activating or repressing the transcription of the target genes (Wang et al. 2015a). Multitudinous TF families have been found in plants, including NAM/ATAF1/CUC2 (NAC), basic leucine zipper (bZIP), APETALA2/ethylene-responsive element (ERE)-binding factor (AP2/ERF), basic helix-loophelix (bHLH), myeloblastosis (MYB), DNA binding with one finger (Dof), and Cys2 (C2) His2type zinc fingers (Yamasaki et al. 2013).

The Dof proteins are a family of plant-specific TFs containing a highly conserved 50- to 56amino acid Dof DNA-binding domain in the N-terminal region (Gupta et al. 2015). The Dof 
domain is structured as a Cys $2 / \mathrm{Cys} 2 \mathrm{Zn}^{2+}$ finger structure that regulates both DNA-protein and protein-protein interactions (Yanagisawa 2002), and recognizes the specific cis-element of (AT)/AAAG in their target gene promoter region, except for a pumpkin Dof protein that binds to an AGTA motif (Diaz et al. 2002; Yanagisawa 2002). The C-terminal of Dof proteins contains a transcriptional regulation domain that can interact with various regulatory proteins and activate the expression of the target genes (Ma et al. 2015).

Dof TFs are involved in many plant-specific physiological processes, including light responsiveness, seed maturation or germination, tissue differentiation, phytochrome and metabolic regulation (Cheng et al. 2018; Gupta et al. 2015; Noguero et al. 2013; Zang et al. 2017). For example, a previous study showed that the Dof protein DOF AFFECTING GERMINATION (DAG2) was a positive regulator of the light-mediated seed germination process in Arabidopsis (Santopolo et al. 2015). The RNA-seq analysis indicated that the $D A G 1$ promoted hypocotyl elongation via regulating the $\mathrm{ABA}$, ethylene, and auxin signals (Lorrai et al. 2018). The overexpression lines of SCAP1 (a Dof transcription factor) increased the number of guard cells (GCs) and protodermal cells recruited in the GC lineage and altered GC distribution and spacing patterns, indicating that SCAP1 could integrate different aspects of GC biology, including specification, spacing, and maturation (Castorina et al. 2016). SlDof10 regulated the vascular tissue formation during ovary development in tomatoes (Rojas-Gracia et al. 2019). The overexpression of AtDOF5.4/OBP4 in Arabidopsis reduced the cell size and number and resulted in dwarf plants, which strongly suggested that $O B P 4$ was a negative regulator of cell cycle progression and cell growth (Xu et al. 2016b). A PpDof5 transcription factor in Pinus pinaster played a vital role in controlling ammonium assimilation for glutamine biosynthesis in conifers (Rueda-Lopez et al. 2008). Furthermore, the overexpression of PpDof5 exhibited higher growth in transgenic hybrid poplars than in controls, enhanced the capacity for inorganic nitrogen uptake, and caused significantly increased accumulation of carbohydrates (Rueda-Lopez et al. 2017). 
84 Some studies showed Dof TFs play important roles in response to biotic and abiotic stresses in

85 plants (Zang et al. 2017). The gain- or loss-of-function analysis of SlDof22 in tomatoes showed

86 that SlDof22 affected ascorbate accumulation and enhanced the tolerance to salinity in plants

87 (Cai et al. 2016). The GhDofl of Gossypium hirsutum improved salt and cold tolerance and seed

88 oil content in transgenic cotton (Su et al. 2017). The OsDof15-mediated ethylene biosynthesis

89 played an important role in inhibiting primary root elongation by salt stress in rice (Qin et al.

90 2019). MdDof54-overexpressing plants had higher photosynthesis rates and shoot hydraulic

91 conductivity under long-term drought stress and higher survival percentages under short-term

92 drought stress compared with nontransgenic plants, illustrating that MdDof54 could improve the

93 drought resistance (Chen et al. 2020b).

94 Although Dofs have been investigated in diverse biological processes, their functional roles and

95 regulatory mechanisms remain unclear. In addition, many members of the Dof family have not

96 yet been characterized, especially in woody plants. Birch (Betula platyphylla) is a valuable

97 broad-leaved pioneer tree of eastern Asia. It is important to stabilize forest ecosystems and

98 regeneration. Birch is also widely used in architecture, furniture, and paper production (Kitao et

99 al. 2001; Pamidi et al. 2020; Xing \& Liu 2012). In this study, 26 Dof genes were isolated from

100 birch and identified to characterize the sequence and expression patterns of the birch Dof genes,

101 followed by multiple sequence alignment, phylogenetic analysis, conserved motif identification,

102 gene structure characterization, and cis-regulatory element analysis. The expression patterns of

103 the 26 birch Dof genes were analyzed in roots, stems, and leaves at different times under drought

104 stress. The Dof genes significantly up-regulated under drought stress were selected to examine

105 the drought stress tolerance in transgenic plants. The results of this study will provide useful

106 information for further investigation of the functional and regulatory mechanisms of these Dof

107 genes in resistance to abiotic stress in birch.

\section{Materials \& Methods}


110 The assembled birch genome (Chen et al. 2021) was analyzed, and the unigenes were searched

111 using BLASTX against the NR and Swiss-Prot databases for functional annotation (Camacho et

112 al. 2009). All BpDofs (Table S1) were detected from the birch genome by employing a hidden

113 Markov model (HMM) profile of the Dof domain (PF02701) obtained from Pfam

114 (http://pfam.xfam.org) using the HMMER3.0 program (http://hmmer.janelia.org) (Finn et al.

115 2016). The conserved domains of these putative BpDof proteins were identified by searching

116 against the NCBI's conserved domain database (https://www.ncbi.nlm.

117 nih.gov/Structure/cdd/wrpsb.cgi?) (Marchler-Bauer et al. 2015). Predictions of theoretical

118 molecular size, isoelectric point (pI), and other physicochemical properties were conducted using

119 the ExPASy ProtParam tool (http://www.expasy.org/tools/protparam.html) (Gasteiger et al.

120 2003). Nuclear localization signals and transmembrane domains were predicted using SubLoc

121 (http://cello.life.nctu.edu.tw/cello2go/) and TMHMM server 2.0

122 (http://www.cbs.dtu.dk/services/TMHMM/), respectively (Chen et al. 2006; Krogh et al. 2001).

\section{Sequence alignment and phylogenetic analysis of the 26 Dof proteins}

124 The multiple sequence alignments of 26 birch Dof proteins were arrayed in the ClustalW2 of

125 BioEdit7.2.1 software (http://www.ebi.ac.uk/Tools/clustalw2/) (Larkin et al. 2007). A

126 phylogenetic tree of 26 birch Dof proteins with the 39 Dof proteins of Arabidopsis (Table S2)

127 was constructed using MEGA7.0 by the neighbor-joining (NJ) method (Kumar et al. 2016;

128 Tamura et al. 2011). The phylogenetic relationships of 26 birch Dof proteins were analyzed.

\section{Gene structure and conserved motif analysis}

130 The genome sequences of the 26 Dof genes were acquired from the birch genome, and their

131 exon/intron structures were analyzed using the GENE Structure Display Serve 2.0 (http://

132 gsds.cbi.pku.edu.cn/) (Hu et al. 2015). Their conserved motifs with default parameters were 
133 analyzed using MEME (http://meme-suite.org/tools/meme) (Bailey et al. 2009), but the

134 maximum number of motifs was set as 15 .

\section{Chromosomal distribution and synteny analysis}

136 The length of each chromosome and the location of each BpDof gene (Table 1) were retrieved

137 from the birch genome, and the chromosomal distribution of BpDof genes was visualized with

138 the Amazing Super Circos software in TBtools (Chen et al. 2020a). One-step MCScanX

139 SuperFast software in TBtools was used for gene synteny and collinearity analyses with default

140 parameters, and the syntenic map was constructed with the chromosomal locations of BpDofs.

141 Furthermore, to analyze the selection pressure of $B p D o f \mathrm{~s}$, the non-synonymous rate (Ka),

142 synonymous rate (Ks), and $\mathrm{Ka} / \mathrm{Ks}$ values of the corresponding BpDofs were calculated using

143 KaKs Calculator v2.0 (Wang et al. 2009).

\section{Cis-element analysis in the promoters of 26 BpDofs}

145 The 2000-bp length of the upstream DNA sequence with the 5'-untranslated region (UTR) for

146 each Dof gene was obtained from the birch genome. The cis-elements in the promoter sequences

147 of the 26 BpDof genes were analyzed using the PlantCARE database

148 (http://bioinformatics.psb.ugent.be/webtools/plantcare/html/)(Lescot et al. 2002).

\section{Plant materials and drought stress treatment}

150 Birch seeds were placed in a glass bottle loosely wrapped with gauze and rinsed with water for 3

151 days. After filtering out most of the water, the seeds were evenly spread on the soil

152 (soil:vermiculite:perlite = 3:1:1). A thin layer of soil was applied to cover the seeds, after which

153 they were watered and sealed using preservative film with some holes in it. The film was

154 removed from the seeds after germination. Once the seedlings grew to $3-4 \mathrm{~cm}$, the seedlings

155 with uniform growth and in good conditions were selected. One birch seedling was planted in

156 one pot. After about 2 months cultivation, the healthy birch seedlings about $25 \mathrm{~cm}$ height with 
157 similar growth conditions were treated using 20\% PEG6000 for 0.5, 1, 3, 6, 12, and $24 \mathrm{~h}$ in a

158 reversed time order. The control plants were treated with fresh water for $24 \mathrm{~h}$. Each birch

159 seedling was watered with 1 L of 20\% PEG6000 or water. The developing roots, stems, and

160 leaves of the birch seedlings under drought treatments were collected after $24 \mathrm{~h}$. All samples

161 were immediately frozen in liquid nitrogen and stored at $-80^{\circ} \mathrm{C}$. Three biological replicates were

162 conducted in each experiment.

\section{RNA isolation and real-time PCR validation}

164 The RNA of each sample was extracted using a Universal Plant RNA Extraction Kit (BioTeke

165 Corporation, China) from $100 \mathrm{mg}$ plant tissues (roots, stems, or leaves) of birch plants, and

166 cDNA was synthesized from approximately $1 \mu \mathrm{g}$ total RNA using PrimeScript IV First-Strand

167 cDNA Synthesis Mix (TaKaRa, Japan). A 20- $\mu \mathrm{L}$ volume containing $10 \mu \mathrm{L}$ of SYBR Green

168 Real-time PCR Master Mix (BioTake Corporation, China), $1 \mu \mathrm{L}$ of cDNA template, and $1 \mu \mathrm{L}$

169 each of the forward primer $(10 \mu \mathrm{M})$ and reverse primer $(10 \mu \mathrm{M})$ was used (all primers and

170 amplicon sizes are shown in Table S3), after which ultrapure $\mathrm{H}_{2} \mathrm{O}$ was used to make up the

171 reaction volume. The amplicons were completed as follows: $94^{\circ} \mathrm{C}$ for $30 \mathrm{~s} ; 94^{\circ} \mathrm{C}$ for $12 \mathrm{~s}, 58^{\circ} \mathrm{C}$

172 for $30 \mathrm{~s}$, and $72^{\circ} \mathrm{C}$ for $45 \mathrm{~s}$, for $45 \mathrm{cycles}$, followed by $79^{\circ} \mathrm{C}$ for $1 \mathrm{~s}$ for plate reading using an

173 qTOWER ${ }^{3} \mathrm{G}$, analytik Jena AG, Germany. After the final PCR cycle, the temperature of $0.5^{\circ} \mathrm{C}$

174 per second was increased from $55^{\circ} \mathrm{C}$ to $99^{\circ} \mathrm{C}$ to generate the melting curve for samples. The

175 relative mRNA levels were determined by normalizing the PCR threshold cycle number of each

176 gene to that of ubiquitin (GenBank number: FG065618) and $\alpha$-tubulin (GenBank number:

177 FG067376) as internal references for all treatments. The threshold for the Ct values was the

178 machine setting, and the average $\mathrm{Ct}$ value was calculated using three biological replicates. The

179 relative expression levels of the 26 BpDof genes were calculated from the threshold cycle by the

180 delta-delta CT method (Pfaffl et al. 2002).

\section{Vector construction and transient transformation}


182 The full-length coding sequences (CDSs) of BpDof4, BpDof11, and BpDof17 were amplified by

183 PCR, and then constructed into the pROKII vector digested with Sma I (NEB, USA) using the In-

184 Fusion $^{\mathrm{TM}}$ CF Liquid PCR Cloning kit (Takara, Japan) under control of the CaMV 35S promoter

185 for overexpression of BpDof4, BpDof11, and BpDof17 (35S:BpDof), respectively. The primers

186 used for PCR are shown in Supplemental Table S4. The pROKII-35S::BpDof4, 35S::BpDof11,

187 and 35S::BpDof17 were separately transformed into 4-week-old birch seedlings by

188 Agrobacterium tumefaciens-mediated transient expression (Zhang et al. 2012) with some

189 modifications. In brief, Luria-Bertani (LB) liquid medium supplied with $50 \mathrm{mg} / \mathrm{L}$ kanamycin and

$19050 \mathrm{mg} / \mathrm{L}$ rifampicin was used to culture the $A$. tumefaciens strain EHA105 transformed with

191 pROKII-35S::BpDof4, pROKII-35S::BpDof1 1, pROKII-35S::BpDof17, or the empty pROKII-

$19235 \mathrm{~S}$ vector. A. tumefaciens cultures were re-suspended in the transformation solution (1/2 MS +

193 sucrose $[2.0 \%, \mathrm{w} / \mathrm{v}]+10 \mathrm{mM} \mathrm{CaCl}_{2}+120 \mu \mathrm{M}$ acetosyringone $+200 \mathrm{mg} / \mathrm{L}$ DTT + Tween-20

$194[0.02 \%, \mathrm{v} / \mathrm{v}], \mathrm{pH} 5.8]$, which were then harvested at an $\mathrm{OD}_{600}$ of 0.6 by centrifugating at $3000 \mathrm{~g}$

195 for $10 \mathrm{~min}$. For transient genetic transformation, the plants were soaked into the transformation

196 solution and shaken at $120 \mathrm{rpm}$ and $25^{\circ} \mathrm{C}$ for $2 \mathrm{~h}$. Then the plants were planted vertically on $1 / 2$

197 MS solid medium (1/2 MS + sucrose $[2.0 \%, \mathrm{w} / \mathrm{v}]+120 \mu \mathrm{M}$ acetosyringone $+200 \mathrm{mg} / \mathrm{L}$ DTT,

$198 \mathrm{pH}$ 5.8) and incubated at $25^{\circ} \mathrm{C}$ in the dark. After culturing for $48 \mathrm{~h}$, the plants were assumed to

199 have been transformed and were then used for subsequent experiments.

200 Stress tolerance analysis of BpDof4-, BpDof11-, and BpDof17-overexpressing plants

201 The birch plants overexpressing BpDof4, BpDof11, or BpDof17 were treated with 20\% PEG6000

202 for $6 \mathrm{~h}$. The pROKII-35S transformants and the wild-type (WT) birch seedlings were also treated 203 with PEG6000. Water treatment was used as control. The detached leaves of birch plants were 204 incubated with $0.5 \mathrm{mg} / \mathrm{mL}$ nitroblue tetrazolium (NBT, dissolved in phosphate buffer, $\mathrm{pH} 7.8$ ) 205 and $1.0 \mathrm{mg} / \mathrm{mL} \mathrm{3'-diaminobenzidine} \mathrm{(DAB,} \mathrm{dissolved} \mathrm{in} \mathrm{phosphate} \mathrm{buffer,} \mathrm{pH} 3.8$ ) as described 206 previously (Zhang et al. 2011). Evans blue (1.0 mg/mL, dissolved in sterile deionize water) 
207 staining was performed to detect cell death following the published protocols (Kim et al. 2003).

208 Superoxide dismutase (SOD) and peroxidase (POD) activities, $\mathrm{H}_{2} \mathrm{O}_{2}$ content, and electrolyte

209 leakage were measured as previously described (Liu et al. 2015; Wang et al. 2015b). Three

210 independent biological replicates were performed.

\section{Statistical analysis}

212 All statistical analyses were performed using SPSS software (IBM, IL, USA), and ANOVA was 213 used to determine statistically significant differences between results. The level of significance 214 was set at $P<0.05$.

\section{Results}

\section{Identification and characterization of the 26 Dofs in B. platyphylla}

217 Twenty-six full-length Dof TFs (GenBank accession numbers: MW538484-538509) were

218 identified in B. platyphylla (Table 1) using the HMMER3.0 program with a HMM profile of the 219 Dof domain (PF02701), and conserved domains of these putative BpDof proteins were identified 220 by searching against the NCBI's conserved domain database. These proteins encoded by BpDof 221 genes consisted of 160-562 amino acids (aa). The molecular sizes and pI values of these proteins 222 ranged from $17.8 \mathrm{kDa}$ to $61.0 \mathrm{kDa}$ and 4.97 to 9.36 , respectively, and their aliphatic indexes 223 were between 43.34 and 62.44. Analyses of instability indexes and grand average of 224 hydropathicities indicated that most BpDof proteins were unstable hydrophilic proteins except 225 BpDof16 and BpDof20. The charge results showed that BpDof8 and BpDof14 were neutral; 226 BpDof2, BpDof3, BpDof4, BpDof5, BpDof21, BpDof25, and BpDof26 were negative; and the 227 other 17 BpDofs were positive. All BpDof proteins were predicted to be localized to the nucleus. 228 The transmembrane domain analysis indicated that the 26 BpDof proteins did not have $\alpha$-helical 229 transmembrane motifs (Tables S5 and S6). 
230 Sequence alignment and phylogenetic analysis of BpDof proteins

231 The multiple sequence alignments of the 26 birch BpDof proteins (Table S1), together with

232 several representative Dof proteins selected from the published Arabidopsis databases

233 (https://www.arabidopsis.org) and Populus trichocarpa v3.0 genomics resource

234 (https://phytozome.jgi.doe.gov/pz/portal.html\#!info?alias=Org_Ptrichocarpa) (Table S7), were

235 analyzed. The single Dof domain with the $\mathrm{C}(\mathrm{x}) 2 \mathrm{C}(\mathrm{x}) 21 \mathrm{C}(\mathrm{x}) 2 \mathrm{C}$ zinc finger pattern was harbored

236 in the N-terminal region of all the putative 26 BpDofs (Fig. 1).

237 The phylogenetic relationships of the 26 BpDof protein sequences, along with the AtDof protein

238 sequences in A. thaliana, were examined using the NJ method. Results showed that the 26

239 BpDof proteins were classified into four major groups (A to D) (Fig. 2). The subfamily D1 in

240 Group D was the largest group, which included seven members accounting for $26.9 \%$ of all

241 BpDof proteins. The subfamily B1 consisted of five members, accounting for $19.2 \%$. The

242 subfamily C2.1 comprised four members, accounting for 15.4\%. Group A and subfamilies C1,

243 C2.2, and D2 contained two members accounting for 7.7\%, respectively. Subfamilies B2 and C3

244 with only one member had a proportion of $3.8 \%$, respectively. 


\section{Conserved motifs and gene structure analysis}

246 Conserved motifs were identified using the MEME tool, and an unrooted phylogenetic tree was

247 constructed based on BpDof protein sequences (Fig. 3a). Different numbers of conserved motifs

248 were set in MEME so as to find the most significant conserved motifs in the 26 BpDof proteins

249 based on the statistical significance $\left(E\right.$ value $\left.<10^{-5}\right)$. The results indicated a total of 15 conserved

250 motifs, and the 26 BpDof proteins were classified into four main groups (A-D) including nine

251 subfamilies basing on the phylogenetic tree. Among them, motif 1 and motif 2 were common

252 motifs shared in almost all BpDof proteins except that BpDof20 lacked motif 2, implying that

253 they were conserved motifs. Some of the BpDof proteins possessed specific motifs, for example,

254 motif 7 was only present in BpDof3, BpDof14, BpDof17, BpDof21, and BpDof26, and motif 11

255 and motif 13 were only distributed in BpDof6, BpDof7, and BpDof22, suggesting these motifs

256 may be relevant to various functions of $B p D$ of genes.

257 The transcript sequences of 26 BpDofs were compared with genomic sequences to obtain the 258 distribution of introns and exons (Fig. 3b). The number of introns in the 26 BpDofs ranged from

2590 to 2 . As a result, 12 BpDof genes contained only exons without any introns (BpDofl, BpDof2, 260 BpDof4, BpDof5, BpDof8, BpDof11, BpDof12, BpDof13, BpDof15-BpDof18), 9 BpDof genes

261 (BpDof6, BpDof7, BpDof14, BpDof19-BpDof22, BpDof24 and BpDof25) respectively contained 262 one intron and two exons, whereas 5 BpDof genes (BpDof3, BpDof9, BpDof10, BpDof23 and 263 BpDof26) contained two introns and three exons.

\section{Chromosomal distribution and inter-specific synteny analysis of BpDofs}

265 We mapped the birch Dof family genes on birch chromosomes to obtain their chromosomal 266 distribution. The results (Fig. 4) showed that the 26 BpDofs were unevenly distributed on the 14 267 chromosomes of birch genome, with only one BpDof gene located on Chr02, Chr03, Chr10, and 268 Chr13, respectively. Two BpDof genes were located on Chr04 and Chr08, respectively. Three 
BpDof genes were located on Chr07, Chr11, and Chr14, respectively. Four BpDof genes were located on Chr12, and five BpDofs on Chr06.

271 During plant evolution, gene duplication is a universal event in all organisms and is important in 272 dissecting the novelties (Lynch \& Conery 2000). According to Fig. 4, seven duplication events 273 were predicted among six chromosomes (Chr02, Chr04, Chr06, Chr08, Chr11, and Chr14), and 274 these duplication events occurred in three subfamilies (B1, C2.1, and D1) of BpDof genes. All of 275 the potential duplication events were inter-chromosomal duplication that occurred between two 276 different chromosomes. Furthermore, the duplicated genes belonged to the same subfamilies, and

277 the three groups of genes were found to have strong

278 collinearity. BpChr06G02126 and BpChr02G19918 were in one group, BpChr06G09621, 279 BpChr11G09292, and BpChr14G12625 were in another group, and the last group 280 included BpChr04G00494, BpChr08G17028, and BpChr11G05806. The Ka/Ks values of these BpDof genes were all less than 1 except those of BpChr11G09292-BpChr14G12625 (Table 2), indicating that they have undergone strong purifying selection during the evolution. The Ks value of BpChr11G09292-BpChr14G12625 was NaN leading to $\mathrm{Ka} / \mathrm{Ks}$ value as $\mathrm{NaN}$, indicating that gene duplication caused mutation at the nucleic acid level but not at the amino acid level.

\section{Analysis of promoter cis-elements of BpDofs}

The putative cis-elements within the 2000-bp genomic sequences upstream with the 5'- UTR of $26 B p D o f s$ were predicted in the PlantCARE database. The results indicated that some ciselements in 26 BpDof gene promoter regions were identified, including MYB and MBS (MYBbinding site, involved in drought inducibility), TC-rich repeats (defense and stress-responsive element), LTR (low temperature-responsive element), GT1-motif (light-responsive element), GBox (light responsiveness element), GA-motif (light-responsive element), W-Box (WRKYbinding site, involved in abiotic stress responsiveness), ABRE (abscisic acid-responsive

293 element), ERE, and GARE-motif (gibberellin-responsive element) (Fig. 5). 
294 Expression patterns of $\boldsymbol{B p D o f}$ genes in response to drought stresses

295 The 2-month-old uniform seedlings were subjected to stress treatment to explore the change in 296 birch Dof expression levels under drought stress. The relative expression levels of the 26 BpDof 297 genes were analyzed in the roots, stems, and leaves of birch under 20\% PEG6000 treatment 298 compared with the control (water treatment) (Fig.6 and Table S8).

299 In roots, the 26 BpDof genes were differentially expressed under PEG6000-simulated drought 300 stress; most of them were upregulated at nearly all time points, and only a few genes at several

301 time points were slightly downregulated. Compared with the control, the expression of BpDof11 302 and BpDof17 was significantly upregulated by about 4-fold to 199-fold, and about half of $303 B p D o f s$ reached their peak expression at $12 \mathrm{~h}$ after treatment. In stems, the expression of most $304 B p D o f$ s showed no obvious change. The expression of BpDof3 (6-fold) at 12 h, BpDof22 (7-fold) 305 at $12 \mathrm{~h}$, and BpDof24 (7-fold) at $6 \mathrm{~h}$ was slightly upregulated. However, the expression of 306 BpDof5 was largely downregulated by about 26 -fold at $12 \mathrm{~h}$ compared with the control. In

307 leaves, the expression of most genes was significantly different compared with the control at 308 most treatment time points. For example, the expression of BpDof4 (322-fold), BpDof5 (68-fold), 309 and BpDofl 4 (106-fold) peaked at $0.5 \mathrm{~h}$, while that of BpDof17 (59-fold) peaked at $24 \mathrm{~h}$.

310 However, the expression of BpDof 24 was downregulated at all treatment time points compared 311 with the control.

312 Plants overexpressing BpDof4, BpDof11, and BpDof17 had reduced oxidative stress and cell 313 membrane damage

314 The expression of BpDof4, BpDof11, and BpDof17 was markedly upregulated at some times 315 under drought treatment compared with the control. Therefore, these three genes were selected 316 for further exploration. NBT and DAB staining was used to detect $\mathrm{O}^{2-}$ and $\mathrm{H}_{2} \mathrm{O}_{2}$ levels so as to 317 determine whether the drought tolerance was strengthened in the BpDof4-, BpDof11-, and 318 BpDof17- overexpressing plants (Fig. 7). The leaves from BpDof4, BpDof1 1, BpDof17, WT and 
pROKII-35S plants were stained with NBT or DAB; the stained leaves from water-treated plants

320

321

322

323

324

325

326

327

328

329

330

331

332

333

334

335

336

337

338

339

340

341

342

343

344

were used as controls. Under drought treatment, the $\mathrm{O}^{2-}$ and $\mathrm{H}_{2} \mathrm{O}_{2}$ levels in the leaves of BpDof4-, BpDof11-, and BpDof17-overexpressing plants were greatly reduced compared with those in WT and pROKII-35S plants. The contents of $\mathrm{O}^{2-}$ and $\mathrm{H}_{2} \mathrm{O}_{2}$ negatively reflected the reactive oxygen species (ROS) scavenging ability of plants. Therefore, the results indicated that BpDof4-, BpDof11-, and BpDof17-overexpressing plants had enhanced abilities to scavenge ROS. In addition, Evans blue staining was used to detect cell membrane damage. Further, BpDof4-, BpDof11-, and BpDof17-overexpressing plants showed less intense blue staining compared with WT and pROKII-35S plants under drought treatment, suggesting that they had decreased cell death.

\section{Physiological characterization of BpDof4, BpDof11, and BpDof17-overexpressing plants}

In this study, SOD and POD activities, $\mathrm{H}_{2} \mathrm{O}_{2}$ content, and electrolyte leakage were used to assess the resistance of BpDof4-, BpDof11-, and BpDof17-overexpressing plants to drought stress, as well as that of the pROKII-35S transformants and WT birch plants (Fig. 8 and Table S9). Water treatment was used as control. SOD and POD play important roles in removing ROS in plants under stress. Results showed that the SOD activity in BpDof11-overexpressing plants was significantly higher than that in WT and pROKII-35S plants; however, the SOD activity in BpDof4- and BpDof17-overexpressing plants had not obvious change compared with WT and pROKII-35S plants. POD activity was significantly higher in BpDof4-, BpDof11-, and BpDof17overexpressing plants compared with WT and pROKII-35S plants under drought stress. The $\mathrm{H}_{2} \mathrm{O}_{2}$ level decreased by $19.13 \%, 36.12 \%$, and $7.01 \%$, respectively, in BpDof4-, BpDofl1-, and BpDof17-overexpressing plants compared with the control. Cell death was evaluated using the electrolyte leakage rate. Electrolyte leakage showed a slight decrease in BpDof4-, BpDof11-, and BpDof17-overexpressing plants compared with the pROKII-35S and WT plants under drought stress. These results suggested that BpDof4, BpDof11, and BpDof17 could enhance the ROS scavenging ability and inhibit cell death in plants. 
345 Discussion

346 In this study, sequences of the 26 BpDof genes were obtained from the birch genome. All

347 putative BpDof TFs had a single Dof domain with a $C(x) 2 C(x) 21 C(x) 2 C$ zinc finger motif in the

348 N-terminal region (Fig. 1), indicating that they were Dof proteins. The amino acid sequences in

349 the C-terminal transcriptional regulatory domains of the $26 \mathrm{BpDof}$ proteins varied, suggesting

350 that the functions of the 26 BpDof proteins might be diverse (Diaz et al. 2002).

351 Based on the phylogenetic analysis of the A. thaliana Dofs (Yanagisawa 2002; Yu et al. 2020),

352 the birch Dof gene family was divided into four groups including nine subfamilies (group A-D,

353 subfamily A, B1, B2, C1, C2.1, C2.2, C3, D1, and D2) (Fig. 2). Each of the birch Dof genes had

354 one or more homologous genes in Arabidopsis, implying that Dof genes in birch might play

355 similar roles as their homologs in Arabidopsis (Zhou et al. 2020). Motifs 1 and 2 were commonly

356 shared by most BpDof family members (Fig. 3a), which was consistent with the results obtained

357 from Arabidopsis, rice, cucumber, and tomato (Cai et al. 2013; Lijavetzky et al. 2003; Wen et al.

358 2016), thus suggesting that BpDof TFs were evolutionarily conserved in plants. The exon-intron

359 structure of genes can provide insights into the evolutionary relationships within certain gene

360 families (Zhou et al. 2020; Zhou et al. 2018). In the present study, the numbers of introns in

361 BpDof genes ranged from 0 to a maximum of 2, and most BpDof genes contained a single intron

362 or no intron at all (Fig. 3b). Similar results have been reported in many other plant species, such

363 as Arabidopsis (Kushwaha et al. 2011), rice (Lijavetzky et al. 2003), cucumber (Wen et al.

364 2016), poplar (Wang et al. 2017), eggplant (Wei et al. 2018), and pear (Liu et al. 2020),

365 indicating that the exon-intron structure of Dof genes is highly conserved across plant species,

366 which may be related to their similar functions. In a specific gene family, the integration and

367 realignment of gene fragments might result in exon-intron variation (Xu et al. 2016a), and the

368 disparate exon-intron structures of the BpDof genes indicated that they may play different roles. 
369 The putative cis-elements in promoters of 26 BpDofs were analyzed in the PlantCARE database.

370 The results (Fig. 5) showed that MYB, MBS, TC-rich, LTR, GT1-motif, G-Box, GA-motif, W-

371 Box, ABRE, ERE, and GARE-motifs, which were related to drought tolerance (Zhang et al.

372 2019), flavonoid biosynthesis (Wang et al. 2018), defense and stress responsiveness (Zhang et al.

373 2005), low temperature tolerance (Feng et al. 2019), light response (Zhang et al. 2013; Zhu et al.

374 2015), abiotic stress responsiveness (Yan et al. 2019), abscisic acid responsiveness (Choi et al.

375 2005), ethylene responsiveness (Rawat et al. 2005), and gibberellin responsiveness, were found

376 in promoters of the 26 BpDof genes. Among these, MYB, MBS, G-Box, GT1-motif, ABRE, and

377 ERE cis-elements were abundant, thus suggesting that these BpDof genes might be involved in

378 drought tolerance, light response, and ABA- and ethylene-responsive signaling.

379 Under adverse environmental conditions, plants have developed many strategies in response to

380 various abiotic stresses (Ma et al. 2015). Previous studies have indicated that some Dof genes

381 might play essential roles in response to abiotic stress (Gu et al. 2019; Zhao et al. 2019).

382 Furthermore, overexpression of Dofs significantly increased the salinity and drought tolerance in

383 transgenic plants (Cheng et al. 2018; He et al. 2015; Liu et al. 2019). In this study, the expression

384 of most BpDof genes was significantly different among birch roots, stems, and leaves under

385 PEG6000 treatment from 0.5 to 24 h (Fig. 6), which suggested that these BpDof genes could be

386 regulated by drought stress and might play key roles in response to drought stress. Under drought

387 treatment, the expression levels of most BpDof genes were up-regulated in roots; no obvious

388 expression differences were observed in stems, but the expression of most BpDof genes was

389 downregulated in leaves (Fig. 6), suggesting Dof genes were differentially expressed in different

390 birch tissues as reported in other plant species (Gupta et al. 2018; Song et al. 2016). Meanwhile,

391 our results also indicated that the responses of most BpDof genes to drought stress were tissue-

392 specific in birch. The differential expression of the 26 BpDof genes under different treatment

393 time also suggested that the signaling pathways in plant response to drought stress were

394 complex.

PeerJ reviewing PDF | (2021:01:57527:3:0:NEW 9 Jul 2021) 
395 Plants are commonly exposed to various adverse situations, which cause the accumulation of

396 ROS (Wang et al. 2005). Therefore, ROS scavenging is important for plant resistance to various

397 stresses. Two major ROS species $\mathrm{O}^{2-}$ and $\mathrm{H}_{2} \mathrm{O}_{2}$ are important molecules in cells, which are

398 involved in oxidative injuries and stress signaling (Zhang et al. 2011). In this study, NBT and

399 DAB staining showed that the ROS accumulation was lower in BpDof4-, BpDof11-, and

400 BpDof17-overexpressing plants than in WT plants under drought treatment (Fig. 7), which was

401 consistent with the levels of $\mathrm{H}_{2} \mathrm{O}_{2}$ (Fig. 8C). The SOD and POD played vital roles in removing

402 ROS. The SOD and POD activities were significantly higher in BpDof4-, BpDof11-, and

403 BpDof17-overexpressing plants than in WT plants under drought stress (Fig. 8A and 8B). These

404 results showed that $B p D o f 4, B p D o f 11$, and BpDofl 7 could enhance the ROS scavenging ability

405 by improving the SOD and POD activities. The result of Evans blue staining (Fig. 7) was

406 consistent with electrolyte leakage rates (Fig. 8D). The BpDof4-, BpDof11-, and BpDof17-

407 overexpressing plants had significantly less intense Evans blue staining and lower electrolyte

408 leakage rates compared with WT plants. The results indicated that BpDof4, BpDof11, and

409 BpDof17 reduced the cell death to enhance the resistance to stress in plants.

410 Conclusions

411 The comprehensive analysis of Dof transpcrition factors was performed in the genome of birch.

412 A total of 26 BpDof genes encoding Dof transpcrition factors were identified from birch, which

413 were classified into four groups and nine subgroups: A, B1, B2, C1, C2.1, C2.2, C3, D1 and D2.

414 The gene structure, conserved motifs and phylogenetic relationships of 26 Dof genes were

415 analyzed. Almost all of the BpDof proteins contained motif1 and motif2 which were considered

416 as the conserved Dof domains. We also investigated the expression patterns of Dof genes at

417 roots, stems and leaves of birch under drought treatment. Moreover, the resistance of BpDof4,

418 BpDof11 and BpDof17 to drought stress in transient transgenic birch plants was conducted. Our

419 results provide valuable information for further understanding of the regulatory mechanisms of 
420 BpDof transcription factors in response to abiotic stress. 


\section{Referance}

422 Bailey TL, Boden M, Buske FA, Frith M, Grant CE, Clementi L, Ren J, Li WW, and Noble WS.

423

424

425

426

427

428

429

430

431

432

433

434 435

436

437

438

439

440

441

442

443

444

445

446

447

448 2009. MEME SUITE: tools for motif discovery and searching. Nucleic Acids Res 37:W202-W208. 10.1093/nar/gkp335

Borrego-Benjumea A, Carter A, Tucker JR, Yao Z, Xu W, and Badea A. 2020. Genome-Wide Analysis of Gene Expression Provides New Insights into Waterlogging Responses in Barley (Hordeum vulgare L.). Plants (Basel) 9(2):e240. 10.3390/plants9020240

Cai X, Zhang C, Shu W, Ye Z, Li H, and Zhang Y. 2016. The transcription factor SIDof22 involved in ascorbate accumulation and salinity stress in tomato. Biochem Biophys Res Commun 474:736-741. 10.1016/j.bbrc.2016.04.148

Cai X, Zhang Y, Zhang C, Zhang T, Hu T, Ye J, Zhang J, Wang T, Li H, and Ye Z. 2013. Genome-wide analysis of plant-specific Dof transcription factor family in tomato. $J$ Integr Plant Biol 55:552-566. 10.1111/jipb.12043

Camacho C, Coulouris G, Avagyan V, Ma N, Papadopoulos J, Bealer K, and Madden TL. 2009. BLAST plus : architecture and applications. Bmc Bioinformatics 10. Artn 421

$10.1186 / 1471-2105-10-421$

Castorina G, Fox S, Tonelli C, Galbiati M, and Conti L. 2016. A novel role for STOMATAL CARPENTER 1 in stomata patterning. BMC Plant Biol 16:172. 10.1186/s12870-0160851-Z

Chen C, Chen H, Zhang Y, Thomas HR, Frank MH, He Y, and Xia R. 2020a. TBtools: An Integrative Toolkit Developed for Interactive Analyses of Big Biological Data. Mol Plant 13:1194-1202. 10.1016/j.molp.2020.06.009

Chen H, Huang N, and Sun Z. 2006. SubLoc: a server/client suite for protein subcellular location based on SOAP. Bioinformatics 22:376-377. 10.1093/bioinformatics/bti822

Chen P, Yan M, Li L, He J, Zhou S, Li Z, Niu C, Bao C, Zhi F, Ma F, and Guan Q. 2020b. The apple DNA-binding one zinc-finger protein MdDof54 promotes drought resistance. Hortic Res 7:195. 10.1038/s41438-020-00419-5

Chen S, Wang Y, Yu L, Zheng T, Wang S, Yue Z, Jiang J, Kumari S, Zheng C, Tang H, Li J, Li 
449

450

451

452

453

454

455

456

457

458

459

460

461

462

463

464

465

466

467

468

469

470

471

472

473

474

475

476

477

478

Y, Chen J, Zhang W, Kuang H, Robertson JS, Zhao PX, Li H, Shu S, Yordanov YS, Huang H, Goodstein DM, Gai Y, Qi Q, Min J, Xu C, Wang S, Qu GZ, Paterson AH, Sankoff D, Wei H, Liu G, and Yang C. 2021. Genome sequence and evolution of Betula platyphylla. Hortic Res 8:37. 10.1038/s41438-021-00481-7

Cheng Z, Hou D, Liu J, Li X, Xie L, Ma Y, and Gao J. 2018. Characterization of moso bamboo (Phyllostachys edulis) Dof transcription factors in floral development and abiotic stress responses. Genome 61:151-156. 10.1139/gen-2017-0189

Choi HI, Park HJ, Park JH, Kim S, Im MY, Seo HH, Kim YW, Hwang I, and Kim SY. 2005. Arabidopsis calcium-dependent protein kinase AtCPK32 interacts with ABF4, a transcriptional regulator of abscisic acid-responsive gene expression, and modulates its activity. Plant Physiol 139:1750-1761. 10.1104/pp.105.069757

Diaz I, Vicente-Carbajosa J, Abraham Z, Martinez M, Isabel-La Moneda I, and Carbonero P. 2002. The GAMYB protein from barley interacts with the DOF transcription factor BPBF and activates endosperm-specific genes during seed development. Plant J 29:453464. 10.1046/j.0960-7412.2001.01230.x

Feng X, Xu Y, Peng L, Yu X, Zhao Q, Feng S, Zhao Z, Li F, and Hu B. 2019. TaEXPB7-B, a beta-expansin gene involved in low-temperature stress and abscisic acid responses, promotes growth and cold resistance in Arabidopsis thaliana. J Plant Physiol 240:153004. 10.1016/j.jplph.2019.153004

Finn RD, Coggill P, Eberhardt RY, Eddy SR, Mistry J, Mitchell AL, Potter SC, Punta M, Qureshi M, Sangrador-Vegas A, Salazar GA, Tate J, and Bateman A. 2016. The Pfam protein families database: towards a more sustainable future. Nucleic Acids Res 44:D279285. 10.1093/nar/gkv1344

Gasteiger E, Gattiker A, Hoogland C, Ivanyi I, Appel RD, and Bairoch A. 2003. ExPASy: The proteomics server for in-depth protein knowledge and analysis. Nucleic Acids Res 31:3784-3788. 10.1093/nar/gkg563

Gu L, Ma Q, Zhang C, Wang C, Wei H, Wang H, and Yu S. 2019. The Cotton GhWRKY91 Transcription Factor Mediates Leaf Senescence and Responses to Drought Stress in Transgenic Arabidopsis thaliana. Front Plant Sci 10:1352. 10.3389/fpls.2019.01352

Gupta S, Malviya N, Kushwaha H, Nasim J, Bisht NC, Singh VK, and Yadav D. 2015. Insights 
479 into structural and functional diversity of Dof (DNA binding with one finger) transcription factor. Planta 241:549-562. 10.1007/s00425-014-2239-3

Gupta S, Pathak RK, Gupta SM, Gaur VS, Singh NK, and Kumar A. 2018. Identification and molecular characterization of Dof transcription factor gene family preferentially expressed in developing spikes of Eleusine coracana L. 3 Biotech 8:82. 10.1007/s13205017-1068-z

He L, Su C, Wang Y, and Wei Z. 2015. ATDOF5.8 protein is the upstream regulator of ANAC069 and is responsive to abiotic stress. Biochimie 110:17-24. 10.1016/j.biochi.2014.12.017

Hu B, Jin J, Guo AY, Zhang H, Luo J, and Gao G. 2015. GSDS 2.0: an upgraded gene feature visualization server. Bioinformatics 31:1296-1297. 10.1093/bioinformatics/btu817

Kim M, Ahn JW, Jin UH, Choi D, Paek KH, and Pai HS. 2003. Activation of the programmed cell death pathway by inhibition of proteasome function in plants. $\mathrm{J}$ Biol Chem 278:19406-19415. 10.1074/jbc.M210539200

Kitao M, Lei TT, Nakamura T, and Koike T. 2001. Manganese toxicity as indicated by visible foliar symptoms of Japanese white birch (Betula platyphylla var. japonica). Environ Pollut 111:89-94. 10.1016/s0269-7491(99)00332-2

Krogh A, Larsson B, von Heijne G, and Sonnhammer EL. 2001. Predicting transmembrane protein topology with a hidden Markov model: application to complete genomes. $J \mathrm{Mol}$ Biol 305:567-580. 10.1006/jmbi.2000.4315

Kumar S, Stecher G, and Tamura K. 2016. MEGA7: Molecular Evolutionary Genetics Analysis Version 7.0 for Bigger Datasets. Molecular Biology and Evolution 33:1870-1874. 10.1093/molbev/msw054

Kushwaha H, Gupta S, Singh VK, Rastogi S, and Yadav D. 2011. Genome wide identification of Dof transcription factor gene family in sorghum and its comparative phylogenetic analysis with rice and Arabidopsis. Molecular Biology Reports 38:5037-5053. 10.1007/s11033-010-0650-9

Larkin MA, Blackshields G, Brown NP, Chenna R, McGettigan PA, McWilliam H, Valentin F, Wallace IM, Wilm A, Lopez R, Thompson JD, Gibson TJ, and Higgins DG. 2007. 
508

509

510

511

512

513

514

515

516

517

518

519

520

521

522

523

524

525

526

527

528

529

530

531

532

533

534

535

536

Clustal W and Clustal X version 2.0. Bioinformatics 23:2947-2948.

10.1093/bioinformatics/btm404

Lescot M, Dehais P, Thijs G, Marchal K, Moreau Y, Van de Peer Y, Rouze P, and Rombauts S. 2002. PlantCARE, a database of plant cis-acting regulatory elements and a portal to tools for in silico analysis of promoter sequences. Nucleic Acids Res 30:325-327. 10.1093/nar/30.1.325

Lijavetzky D, Carbonero P, and Vicente-Carbajosa J. 2003. Genome-wide comparative phylogenetic analysis of the rice and Arabidopsis Dof gene families. BMC Evol Biol 3:17. 10.1186/1471-2148-3-17

Liu J, Cheng Z, Xie L, Li X, and Gao J. 2019. Multifaceted Role of PheDof12-1 in the Regulation of Flowering Time and Abiotic Stress Responses in Moso Bamboo (Phyllostachys edulis). Int J Mol Sci 20(2):E424. 10.3390/ijms20020424

Liu X, Liu Z, Hao Z, Chen G, Qi K, Zhang H, Jiao H, Wu X, Zhang S, Wu J, and Wang P. 2020. Characterization of Dof family in Pyrus bretschneideri and role of PbDof9.2 in flowering time regulation. Genomics 112:712-720. 10.1016/j.ygeno.2019.05.005

Liu YJ, Ji XY, Nie XG, Qu M, Zheng L, Tan ZL, Zhao HM, Huo L, Liu SN, Zhang B, and Wang YC. 2015. Arabidopsis AtbHLH112 regulates the expression of genes involved in abiotic stress tolerance by binding to their E-box and GCG-box motifs. New Phytologist 207:692-709. 10.1111/nph.13387

Lorrai R, Gandolfi F, Boccaccini A, Ruta V, Possenti M, Tramontano A, Costantino P, Lepore R, and Vittorioso P. 2018. Genome-wide RNA-seq analysis indicates that the DAG1 transcription factor promotes hypocotyl elongation acting on ABA, ethylene and auxin signaling. Sci Rep 8:15895. 10.1038/s41598-018-34256-3

Lynch M, and Conery JS. 2000. The evolutionary fate and consequences of duplicate genes. Science 290:1151-1155. 10.1126/science.290.5494.1151

Ma J, Li MY, Wang F, Tang J, and Xiong AS. 2015. Genome-wide analysis of Dof family transcription factors and their responses to abiotic stresses in Chinese cabbage. $B M C$ Genomics 16:33. 10.1186/s12864-015-1242-9

Marchler-Bauer A, Derbyshire MK, Gonzales NR, Lu S, Chitsaz F, Geer LY, Geer RC, He J, 
537

538

539

540

541

542

543

544

545

546

547

548

549

550

551

552

553

554

555

556

557

558

559

560

561

562

563

564

565

Gwadz M, Hurwitz DI, Lanczycki CJ, Lu F, Marchler GH, Song JS, Thanki N, Wang Z, Yamashita RA, Zhang D, Zheng C, and Bryant SH. 2015. CDD: NCBI's conserved domain database. Nucleic Acids Res 43:D222-226. 10.1093/nar/gku1221

Noguero M, Atif RM, Ochatt S, and Thompson RD. 2013. The role of the DNA-binding One Zinc Finger (DOF) transcription factor family in plants. Plant Sci 209:32-45. 10.1016/j.plantsci.2013.03.016

Pamidi TRK, Johansson O, Lofqvist T, and Shankar V. 2020. Comparison of two different ultrasound reactors for the treatment of cellulose fibers. Ultrason Sonochem 62:104841. 10.1016/j.ultsonch.2019.104841

Pfaffl MW, Horgan GW, and Dempfle L. 2002. Relative expression software tool (REST) for group-wise comparison and statistical analysis of relative expression results in real-time PCR. Nucleic Acids Res 30:e36. 10.1093/nar/30.9.e36

Qin H, Wang J, Chen X, Wang F, Peng P, Zhou Y, Miao Y, Zhang Y, Gao Y, Qi Y, Zhou J, and Huang R. 2019. Rice OsDOF15 contributes to ethylene-inhibited primary root elongation under salt stress. New Phytol 223:798-813. 10.1111/nph.15824

Rawat R, Xu ZF, Yao KM, and Chye ML. 2005. Identification of cis-elements for ethylene and circadian regulation of the Solanum melongena gene encoding cysteine proteinase. Plant Mol Biol 57:629-643. 10.1007/s11103-005-0954-7

Riechmann JL, and Ratcliffe OJ. 2000. A genomic perspective on plant transcription factors. Curr Opin Plant Biol 3:423-434. 10.1016/s1369-5266(00)00107-2

Rojas-Gracia P, Roque E, Medina M, Lopez-Martin MJ, Canas LA, Beltran JP, and GomezMena C. 2019. The DOF Transcription Factor SIDOF10 Regulates Vascular Tissue Formation During Ovary Development in Tomato. Front Plant Sci 10:216. $10.3389 /$ fpls.2019.00216

Rueda-Lopez M, Crespillo R, Canovas FM, and Avila C. 2008. Differential regulation of two glutamine synthetase genes by a single Dof transcription factor. Plant $J$ 56:73-85. 10.1111/j.1365-313X.2008.03573.x

Rueda-Lopez M, Pascual MB, Pallero M, Henao LM, Lasa B, Jauregui I, Aparicio-Tejo PM, Canovas FM, and Avila C. 2017. Overexpression of a pine Dof transcription factor in

PeerJ reviewing PDF | (2021:01:57527:3:0:NEW 9 Jul 2021) 
566

567

568

569

570

571

572

573

574

575

576

577

578

579

580

581

582

583

584

585

586

587

588

589

590

591

592

593

594

hybrid poplars: A comparative study in trees growing under controlled and natural conditions. PLoS One 12:e0174748. 10.1371/journal.pone.0174748

Santopolo S, Boccaccini A, Lorrai R, Ruta V, Capauto D, Minutello E, Serino G, Costantino P, and Vittorioso P. 2015. DOF AFFECTING GERMINATION 2 is a positive regulator of light-mediated seed germination and is repressed by DOF AFFECTING GERMINATION 1. BMC Plant Biol 15:72. 10.1186/s12870-015-0453-1

Song A, Gao T, Li P, Chen S, Guan Z, Wu D, Xin J, Fan Q, Zhao K, and Chen F. 2016. Transcriptome-Wide Identification and Expression Profiling of the DOF Transcription Factor Gene Family in Chrysanthemum morifolium. Front Plant Sci 7:199. 10.3389/fpls.2016.00199

Su Y, Liang W, Liu Z, Wang Y, Zhao Y, Ijaz B, and Hua J. 2017. Overexpression of GhDof1 improved salt and cold tolerance and seed oil content in Gossypium hirsutum. J Plant Physiol 218:222-234. 10.1016/j.jplph.2017.07.017

Tamura K, Peterson D, Peterson N, Stecher G, Nei M, and Kumar S. 2011. MEGA5: molecular evolutionary genetics analysis using maximum likelihood, evolutionary distance, and maximum parsimony methods. Mol Biol Evol 28:2731-2739. 10.1093/molbev/msr121

Wang DP, Wan HL, Zhang S, and Yu J. 2009. Gamma-MYN: a new algorithm for estimating Ka and Ks with consideration of variable substitution rates. Biol Direct 4:20. 10.1186/17456150-4-20

Wang FZ, Wang QB, Kwon SY, Kwak SS, and Su WA. 2005. Enhanced drought tolerance of transgenic rice plants expressing a pea manganese superoxide dismutase. J Plant Physiol 162:465-472. 10.1016/j.jplph.2004.09.009

Wang H, Zhao S, Gao Y, and Yang J. 2017. Characterization of Dof Transcription Factors and Their Responses to Osmotic Stress in Poplar (Populus trichocarpa). PLoS One 12:e0170210. 10.1371/journal.pone.0170210

Wang L, Wang C, Qin L, Liu W, and Wang Y. 2015a. ThERF1 regulates its target genes via binding to a novel cis-acting element in response to salt stress. $J$ Integr Plant Biol 57:838-847. 10.1111/jipb.12335

Wang LQ, Zheng L, Zhang CR, Wang YC, Lu MZ, and Gao CQ. 2015b. ThWRKY4 from

PeerJ reviewing PDF | (2021:01:57527:3:0:NEW 9 Jul 2021) 
595

596

597

598

599

600

601

602

603

604

605

606

607

608

609

610

611

612

613

614

615

616

617

618

619

620

621

622

623

Tamarix hispida Can Form Homodimers and Heterodimers and Is Involved in Abiotic Stress Responses. International Journal of Molecular Sciences 16:27097-27106. 10.3390/ijms 161126009

Wang WL, Wang YX, Li H, Liu ZW, Cui X, and Zhuang J. 2018. Two MYB transcription factors (CsMYB2 and CsMYB26) are involved in flavonoid biosynthesis in tea plant [Camellia sinensis (L.) O. Kuntze]. BMC Plant Biol 18:288. 10.1186/s12870-018-1502-3

Wei Q, Wang W, Hu T, Hu H, Mao W, Zhu Q, and Bao C. 2018. Genome-wide identification and characterization of Dof transcription factors in eggplant (Solanum melongena L.). PeerJ 6:e4481. 10.7717/peerj.4481

Wen CL, Cheng Q, Zhao L, Mao A, Yang J, Yu S, Weng Y, and Xu Y. 2016. Identification and characterisation of Dof transcription factors in the cucumber genome. Sci Rep 6:23072. 10.1038/srep23072

Xing L, and Liu XM. 2012. Characterization of Betula platyphylla gene transcripts associated with early development of male inflorescence. Molecular Biology Reports 39:929-935. 10.1007/s11033-011-0818-y

Xu JN, Xing SS, Cui HR, Chen XS, and Wang XY. 2016a. Genome-wide identification and characterization of the apple (Malus domestica) HECT ubiquitin-protein ligase family and expression analysis of their responsiveness to abiotic stresses. Molecular Genetics and Genomics 291:635-646. 10.1007/s00438-015-1129-0

Xu P, Chen H, Ying L, and Cai W. 2016b. AtDOF5.4/OBP4, a DOF Transcription Factor Gene that Negatively Regulates Cell Cycle Progression and Cell Expansion in Arabidopsis thaliana. Sci Rep 6:27705. 10.1038/srep27705

Yamasaki K, Kigawa T, Seki M, Shinozaki K, and Yokoyama S. 2013. DNA-binding domains of plant-specific transcription factors: structure, function, and evolution. Trends Plant Sci 18:267-276. 10.1016/j.tplants.2012.09.001

Yan H, Li M, Xiong Y, Wu J, Teixeira da Silva JA, and Ma G. 2019. Genome-Wide Characterization, Expression Profile Analysis of WRKY Family Genes in Santalum album and Functional Identification of Their Role in Abiotic Stress. Int J Mol Sci 13:20(22). 10.3390/ijms20225676

Peer] reviewing PDF | (2021:01:57527:3:0:NEW 9 Jul 2021) 
624 Yanagisawa S. 2002. The Dof family of plant transcription factors. Trends Plant Sci 7:555-560.

625

626

627

628

629

630

631

632

633

634

635

636

637

638

639

640

641

642

643

644

645

646

647

648

649

650

651

652

\subsection{6/s1360-1385(02)02362-2}

Yang C, Huang Y, Lv W, Zhang Y, Bhat JA, Kong J, Xing H, Zhao J, and Zhao T. 2020. GmNAC8 acts as a positive regulator in soybean drought stress. Plant Sci 293:110442. 10.1016/j.plantsci.2020.110442

Yu Q, Li C, Zhang J, Tian Y, Wang H, Zhang Y, Zhang Z, Xiang Q, Han X, and Zhang L. 2020. Genome-wide identification and expression analysis of the Dof gene family under drought stress in tea (Camellia sinensis). PeerJ 8:e9269. 10.7717/peerj.9269

Zang D, Wang L, Zhang Y, Zhao H, and Wang Y. 2017. ThDof1.4 and ThZFP1 constitute a transcriptional regulatory cascade involved in salt or osmotic stress in Tamarix hispida. Plant Mol Biol 94:495-507. 10.1007/s11103-017-0620-x

Zhang L, Song Z, Li F, Li X, Ji H, and Yang S. 2019. The specific MYB binding sites bound by TaMYB in the GAPCp2/3 promoters are involved in the drought stress response in wheat. BMC Plant Biol 19:366. 10.1186/s12870-019-1948-y

Zhang LF, Li WF, Han SY, Yang WH, and Qi LW. 2013. cDNA cloning, genomic organization and expression analysis during somatic embryogenesis of the translationally controlled tumor protein (TCTP) gene from Japanese larch (Larix leptolepis). Gene 529:150-158. 10.1016/j.gene.2013.07.076

Zhang W, Ruan J, Ho TH, You Y, Yu T, and Quatrano RS. 2005. Cis-regulatory element based targeted gene finding: genome-wide identification of abscisic acid- and abiotic stressresponsive genes in Arabidopsis thaliana. Bioinformatics 21:3074-3081. 10.1093/bioinformatics/bti490

Zhang X, Wang L, Meng H, Wen HT, Fan YL, and Zhao J. 2011. Maize ABP9 enhances tolerance to multiple stresses in transgenic Arabidopsis by modulating ABA signaling and cellular levels of reactive oxygen species. Plant Molecular Biology 75:365-378. 10.1007/s11103-011-9732-X

Zhang Y, Wang YC, and Wang C. 2012. Gene overexpression and gene silencing in Birch using an Agrobacterium-mediated transient expression system. Molecular Biology Reports 39:5537-5541. 10.1007/s11033-011-1357-2 
653 Zhao MJ, Yin LJ, Liu Y, Ma J, Zheng JC, Lan JH, Fu JD, Chen M, Xu ZS, and Ma YZ. 2019.

654 The ABA-induced soybean ERF transcription factor gene GmERF75 plays a role in

655 enhancing osmotic stress tolerance in Arabidopsis and soybean. BMC Plant Biol 19:506.

$656 \quad 10.1186 / \mathrm{s} 12870-019-2066-6$

657 Zhou Y, Cheng Y, Wan C, Li J, Yang Y, and Chen J. 2020. Genome-wide characterization and

658 expression analysis of the Dof gene family related to abiotic stress in watermelon. PeerJ

$659 \quad 8: e 8358.10 .7717 /$ peerj.8358

660 Zhou Y, Hu L, Jiang L, and Liu S. 2018. Genome-wide identification and expression analysis of

661 YTH domain-containing RNA-binding protein family in cucumber (Cucumis sativus).

662 Genes Genomics 40:579-589. 10.1007/s13258-018-0659-3

663 Zhu X, Chen J, Xie Z, Gao J, Ren G, Gao S, Zhou X, and Kuai B. 2015. Jasmonic acid promotes

664

665 degreening via MYC2/3/4- and ANAC019/055/072-mediated regulation of major chlorophyll catabolic genes. Plant J 84:597-610. 10.1111/tpj.13030

666 


\section{Table $\mathbf{1}$ (on next page)}

Characterization of $26 B p D$ of genes and proteins

Al, Aliphatic index; CR, charged residues ( positively: +; negatively: -; neutral: =); GRAVY, grand average of hydropathicity; II, instability index; MW, protein molecular size (kDa); PI, isoelectric point. 
1 Table 1. Characterization of 26 BpDof transcription factors.

\begin{tabular}{|c|c|c|c|c|c|c|c|c|c|c|}
\hline Name & Locus ID & Chromosome position & Len & MW & PI & AI & II & Stability & GRAVY & CR \\
\hline BpDof1 & BPChr06G16490 & Chr06:3395833:3404094 & 310 & 33.2 & 9.03 & 61.68 & 56.67 & Unstable & -0.525 & + \\
\hline BpDof2 & BPChr12G11401 & Chr12:1468437:1469132 & 231 & 24 & 6.06 & 49.83 & 54.00 & Unstable & -0.436 & - \\
\hline BpDof3 & BPChr11G05806 & Chr11:4202830:4206047 & 562 & 61 & 5.15 & 53.72 & 58.45 & Unstable & -0.79 & - \\
\hline BpDof4 & BPChr12G08354 & Chr12:5473320:5474114 & 264 & 29.4 & 4.97 & 59.47 & 66.08 & Unstable & -0.684 & - \\
\hline BpDof5 & BPChr14G09159 & Chr14:8257436:8258170 & 244 & 27.1 & 6.24 & 55.9 & 54.33 & Unstable & -0.595 & - \\
\hline BpDof6 & BPChr06G09621 & Chr06:36382710:36383854 & 326 & 35.3 & 8.89 & 48.47 & 55.66 & Unstable & -0.771 & + \\
\hline BpDof7 & BPChr14G12625 & Chr14:5137993:5139183 & 340 & 35.6 & 9.36 & 52.06 & 53.49 & Unstable & -0.549 & + \\
\hline BpDof8 & BPChr12G29175 & Chr12:10562079:10563017 & 312 & 33.9 & 7.18 & 57.24 & 57.26 & Unstable & -0.678 & $=$ \\
\hline BpDof9 & BPChr06G29469 & Chr06:6207612:6212636 & 265 & 29.1 & 9.13 & 46.38 & 41.85 & Unstable & -0.829 & + \\
\hline $\begin{array}{l}\text { BpDof1 } \\
0\end{array}$ & BPChr02G19918 & Chr02:22002669:22010046 & 248 & 27.3 & 9.07 & 57.38 & 49.41 & Unstable & -0.676 & + \\
\hline BpDof1 & BPChr10G04282 & Chr10:1350584:1369701 & 295 & 32.8 & 7.61 & 48.27 & 49.33 & Unstable & -0.886 & + \\
\hline
\end{tabular}


BpDof1

2

BpDof1

3

BpDof1

4

BpDof1

5

BpDof1

6

BpDof1

7

BpDof1

8

BpDof1
BPChr06G19208 Chr06:11028826:11029461

BPChr03G28866 Chr03:20524755:20525537

BPChr04G00494 Chr04:8288764:8291483

BPChr04G23864 Chr04:3304076:3305071

331

35.4

9.12

53.66

46.02 Unstable

$-0.644+$

BPChr06G02126 Chr06:1426392:1427096

$\begin{array}{llllll}234 & 24.1 & 8.51 & 50.9 & 37.07 & \text { Stable }\end{array}$

$\begin{array}{llllll}160 & 17.8 & 9.01 & 49.38 & 43.05 & \text { Unstable }\end{array}$

$-0.803+$

BPChr07G09798 Chr07:24560688:24561170

BPChr07G18918 Chr07:19263129:19264016

295

$32.7 \quad 9.15$

40.34

66.71 Unstable

$-1.082+$

BPChr07G18939 Chr07:19281783:19282965 $\begin{array}{llllll}342 & 37.5 & 8.43 & 52.19 & 53.51 & \text { Unstable }\end{array}$

$-0.758+$ 
9

\begin{tabular}{|c|c|c|c|c|c|c|c|c|c|c|}
\hline $\begin{array}{l}\text { BpDof2 } \\
0\end{array}$ & BPChr08G01518 & Chr08:8408149:8408980 & 182 & 20.6 & 8.99 & 51.48 & 33.69 & Stable & -0.866 & + \\
\hline $\begin{array}{l}\text { BpDof2 } \\
1\end{array}$ & BPChr08G17028 & Chr08:39507804:39510785 & 490 & 53.3 & 6.71 & 57.14 & 59.52 & Unstable & -0.773 & - \\
\hline $\begin{array}{l}\text { BpDof2 } \\
2\end{array}$ & BPChr11G09292 & Chr11:23999498:24000624 & 319 & 34.5 & 9.21 & 59.97 & 66.82 & Unstable & -0.643 & + \\
\hline $\begin{array}{l}\text { BpDof2 } \\
3\end{array}$ & BPChr11G10185 & Chr11:35669848:35671309 & 297 & 32.9 & 8.54 & 49.26 & 53.21 & Unstable & -0.795 & + \\
\hline $\begin{array}{l}\text { BpDof2 } \\
4\end{array}$ & BPChr12G29204 & Chr12:9026101:9027218 & 249 & 27 & 9.32 & 47.43 & 50.63 & Unstable & -0.738 & + \\
\hline $\begin{array}{l}\text { BpDof2 } \\
5\end{array}$ & BPChr13G02551 & Chr13:5309999:5313177 & 510 & 55.6 & 6.54 & 52.43 & 57.19 & Unstable & -0.731 & - \\
\hline $\begin{array}{l}\text { BpDof2 } \\
6\end{array}$ & BPChr14G05515 & Chr14:9880786:9891340 & 518 & 57.1 & 6.19 & 61.39 & 52.63 & Unstable & -0.554 & - \\
\hline
\end{tabular}


Table 2 (on next page)

Duplication models for BpDof gene pairs in birch. 
Table 2. Duplication models for BpDof gene pairs in birch.

\begin{tabular}{lccccc}
\hline Duplicate gene pair & Ka & Ks & Ka/Ks & AverageS-sites & AverageN-sites \\
\hline BPChr11G05806- BPChr04G00494 & 0.359251491 & 1.402780691 & 0.256099541 & 320.75 & 1077.25 \\
BPChr11G05806- BPChr08G17028 & 0.257338858 & 1.155021891 & 0.222799984 & 333.9166667 & 1112.083333 \\
BPChr04G00494- BPChr08G17028 & 0.303409975 & 1.775006855 & 0.170934537 & 310.0833333 & 1042.916667 \\
BPChr06G09621- BPChr11G09292 & 0.342798859 & 2.485336412 & 0.137928554 & 206.1666667 & 657.8333333 \\
BPChr06G09621- BPChr14G12625 & 0.318070599 & 1.25186786 & 0.254076815 & 220.4166667 & 697.5833333 \\
BPChr11G09292- BPChr14G12625 & 0.398755789 & NaN & NaN & 205.8333333 & 664.1666667 \\
BPChr06G02126- BPChr02G19918 & 0.683281032 & 3.032288451 & 0.225335104 & 152 \\
\hline
\end{tabular}




\section{Figure 1}

Identification of conserved Dof subdomains in 26 BpDofs.

Multiple sequence alignments of 26 BpDofs from birch with 2 AtDofs from Arabidopsis and 2 PtDofs from popular were obtained using ClustalW in the BioEdit software. The zinc finger structure was displayed under multiple sequence alignment results, and the font size in the zinc finger structure represented the frequency of the respective amino acid.

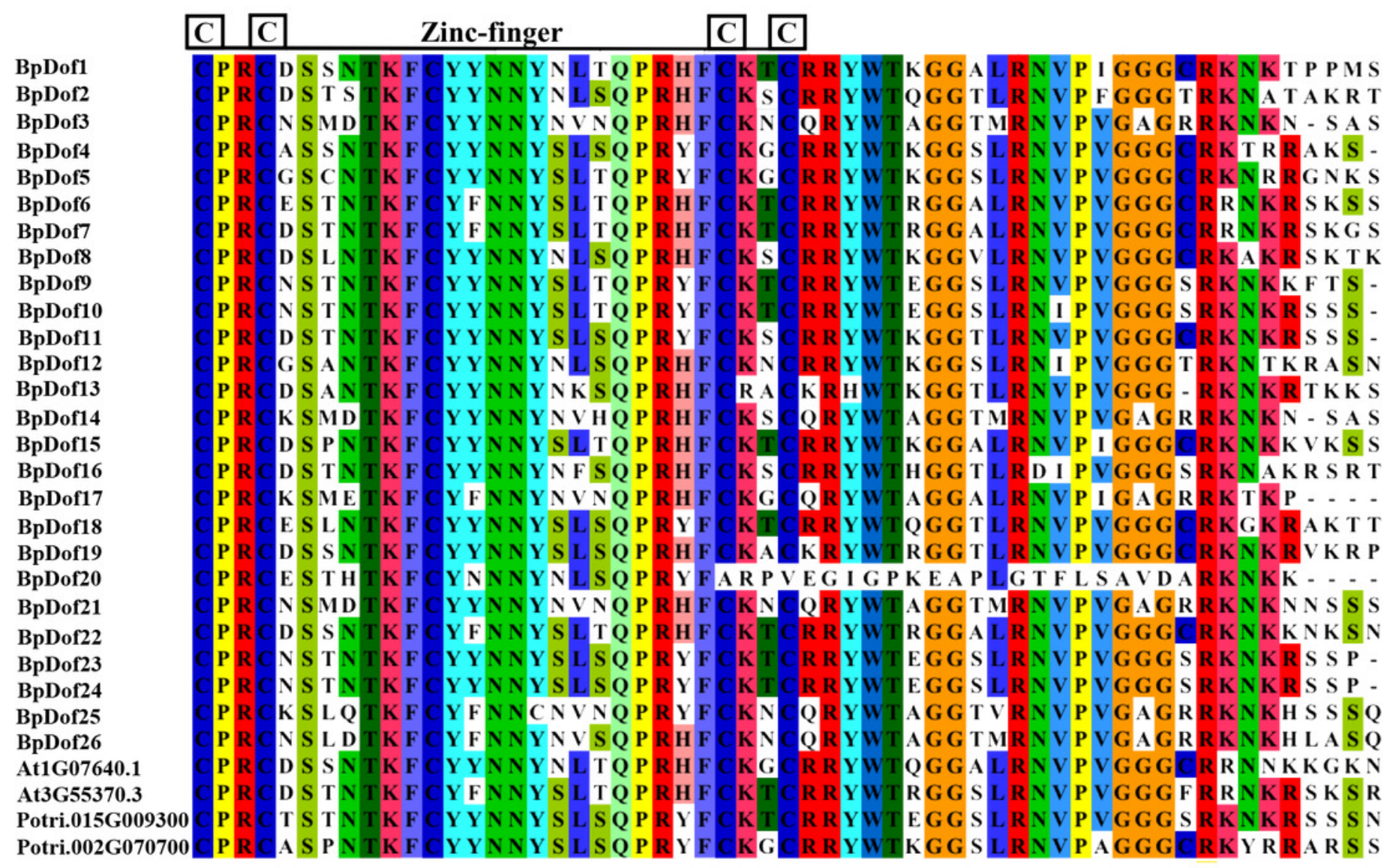
$\frac{\text { CRC.S.NTKFCYNNY L.PR FCK.CRRVWT,GG.LRNVPGGG.RKNK, }}{\text { CPRCDSTNTKFCYYNNYSSQPRHFCKTCRRYWTKGGTLRNVPVGGGCRKKRSKSS }}$ 
Figure 2

Phylogenetic analysis of 26 BpDof protein sequences in birch with AtDof protein sequences in Arabidopsis.

The phylogenetic analysis of 26 BpDof protein sequences in birch and 39 AtDofs protein sequences in Arabidopsis was performed using MEGA 7.0. The full-length amino acid sequences of all Dof proteins were aligned using ClustalX 1.83. The star in the black circle represents BpDofs. 


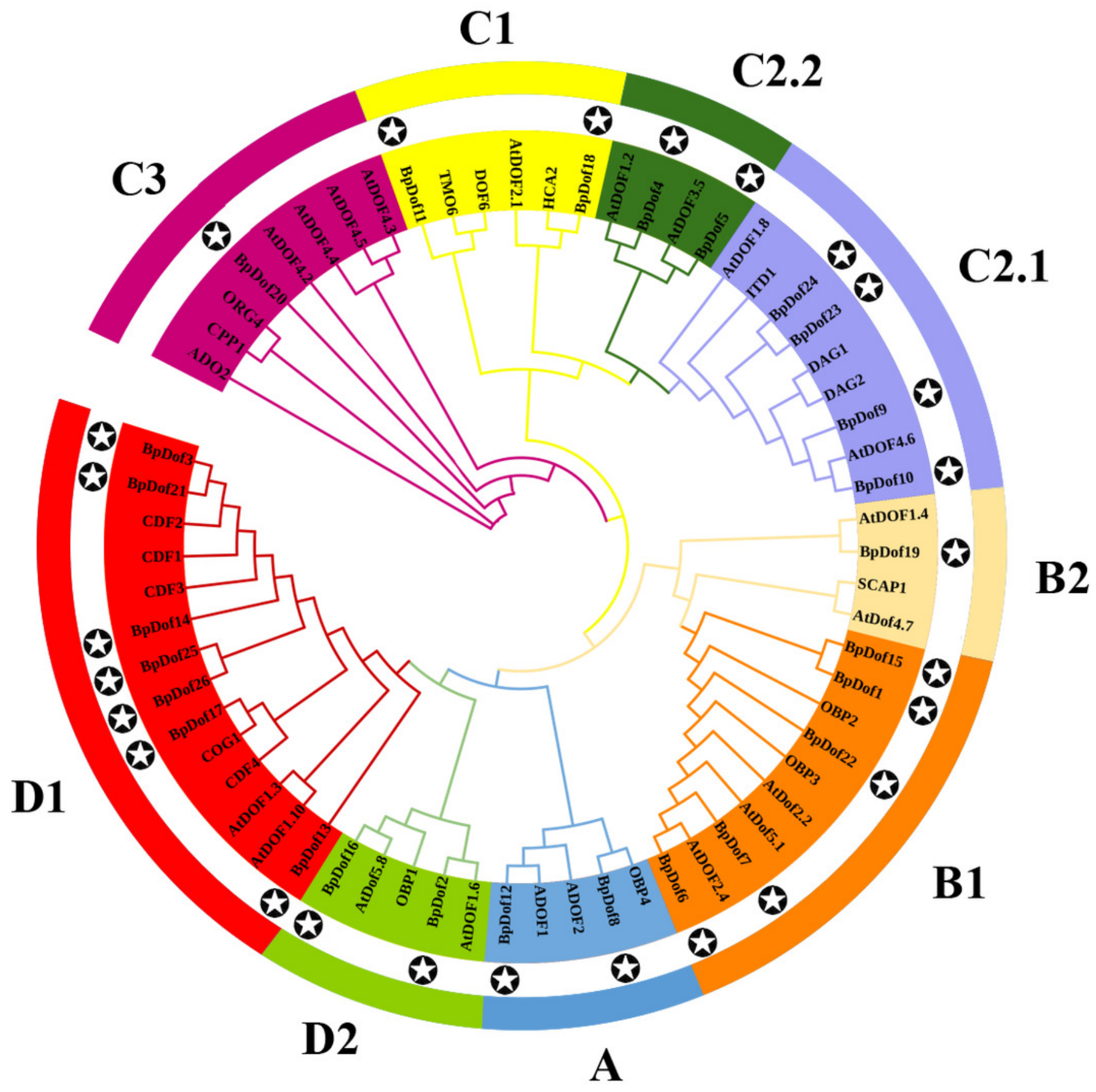


Figure 3

The Motif and gene structure of the Dof protein family in birch.

(a) The distribution of motifs in BpDof proteins was examined. The 15 conserved motifs ( $E$ value $<10^{-5}$ ) in birch are represented with different color boxes, and the motif sequence logos are displayed in the upper right corner. The dark gray line shows the length of proteins. (b) Intron-exon patterns of 26 BpDof genes from birch. The legend was showed by the side of the motif sequence logos.

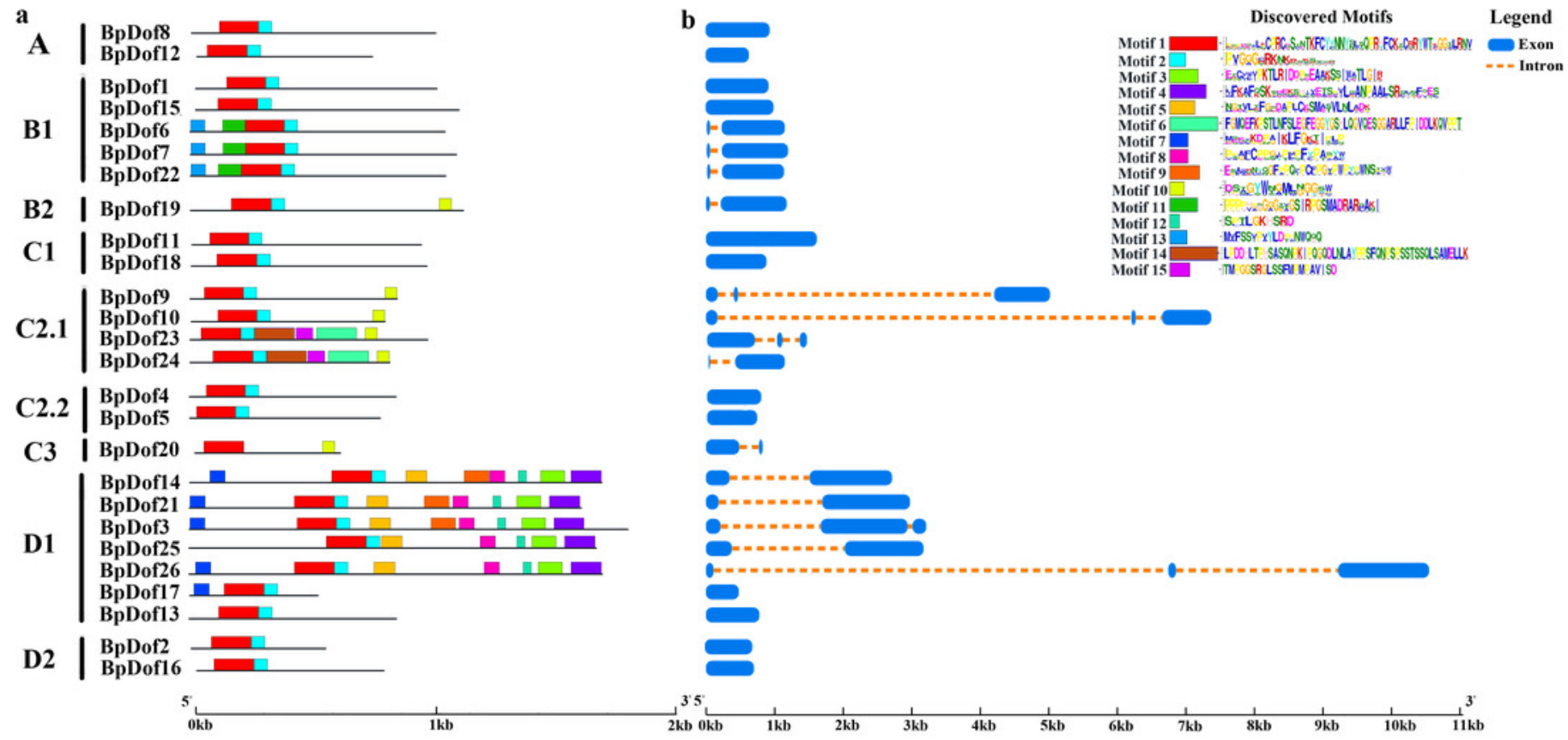




\section{Figure 4}

Chromosomal distribution and interchromosomal relationships of $26 \mathrm{BpDof}$ genes.

Grey lines indicate all synteny blocks in the birch genome, and the red lines represent duplicated BpDof gene pairs. The thermal maps in the orange rectangles represent the gene density on the chromosomes. Grey rectangles represent birch chromosomes 01-14 and the chromosome number was displayed in the grey recetangles. 


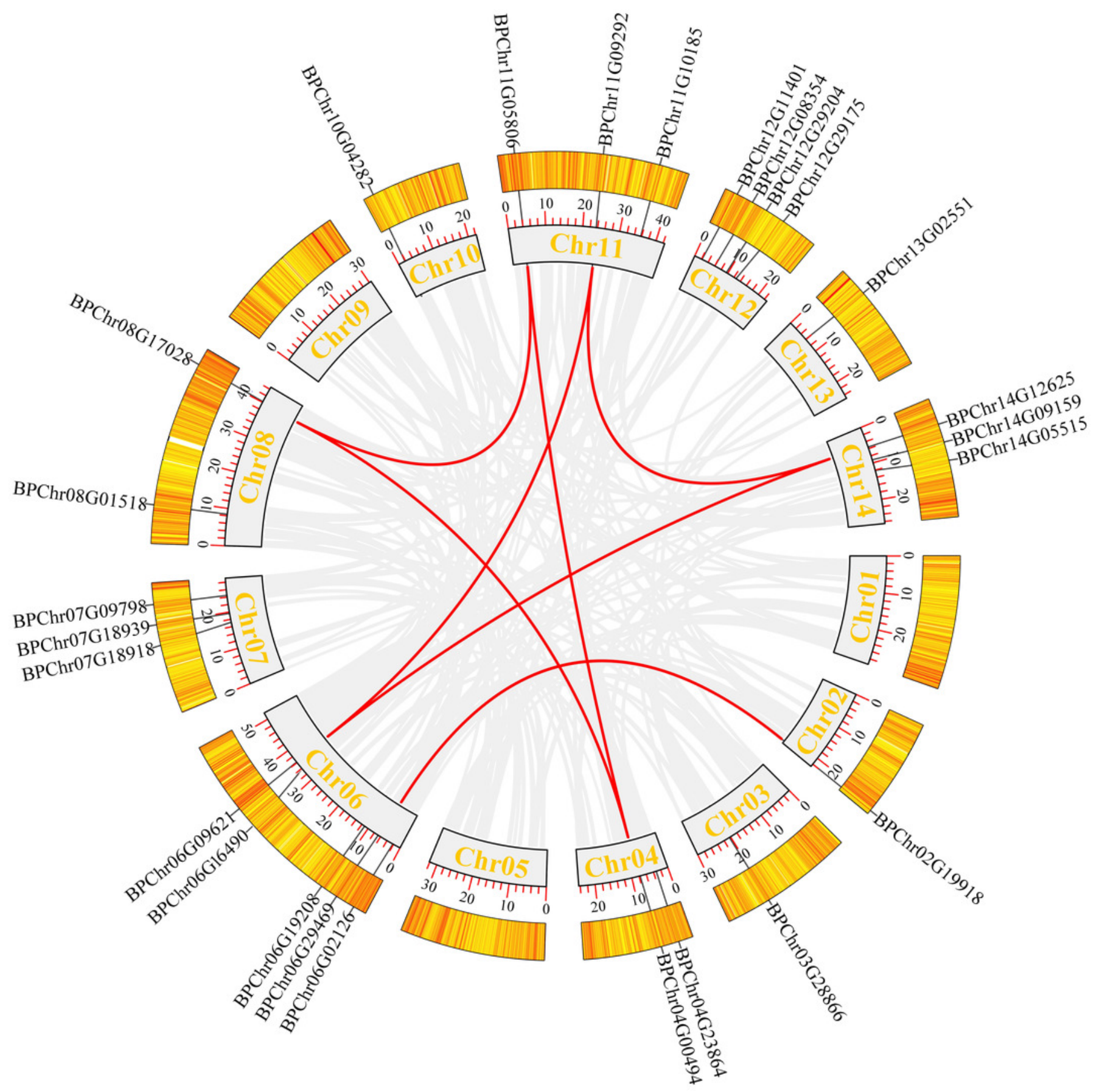


Figure 5

Distribution of cis-elements in the promoters of 26 BpDof genes.

The 11 different cis-elements in the promoters of the $26 \mathrm{BpDof}$ genes are represented in different color boxes.

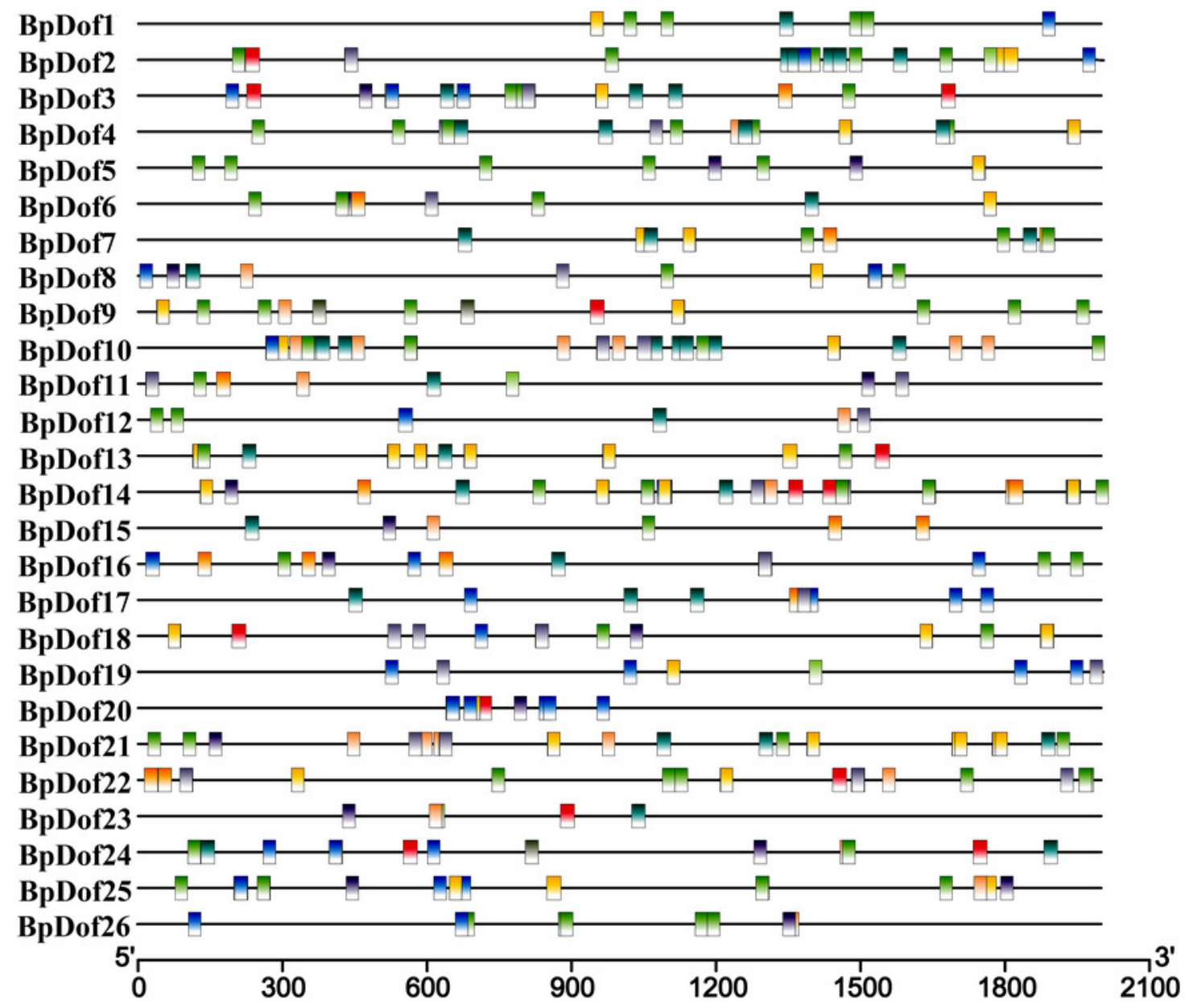

MYB

ABRE

G-Box

ERE

TC-rich repeats GT1-motif MBS

GA-motif

LTR

W-box

GARE-motif 


\section{Figure 6}

Expression patterns of the 26 BpDof genes in the roots, stems, and leaves of 2-monthold B. platyphylla seedlings under drought stress (20\% PEG6000) treatment.

The gene expression of different tissues of birch plants was analyzed by qRT-PCR. The expression levels of the 26 BpDof genes after 0-h treatment was used as the control to detect the relative expression levels of the genes.All ratios are $\log 2$ transformed so that inductions and repressions of identical magnitude are numerically equal but opposite in sign. Log ratios of 0 (ratios of 1 ) are colored yellow, and increasingly positive (induction) or negative (repression) log ratios are colored red or blue with increasing intensity, respectively. Red means induction and blue repression in arrays.

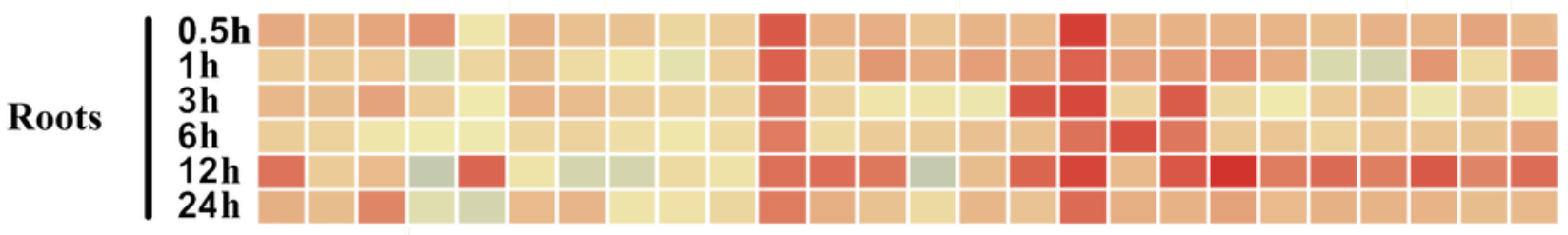

$10.5 \mathrm{~h}$

Stems

$1 \mathrm{~h}$
$3 \mathrm{~h}$
$6 \mathrm{~h}$
$12 \mathrm{~h}$
$24 \mathrm{~h}$

\begin{tabular}{l|l} 
Leaves & $\begin{array}{l}0.5 \mathrm{~h} \\
1 \mathrm{~h} \\
3 \mathrm{~h} \\
6 \mathrm{~h} \\
12 \mathrm{~h} \\
24 \mathrm{~h}\end{array}$
\end{tabular}

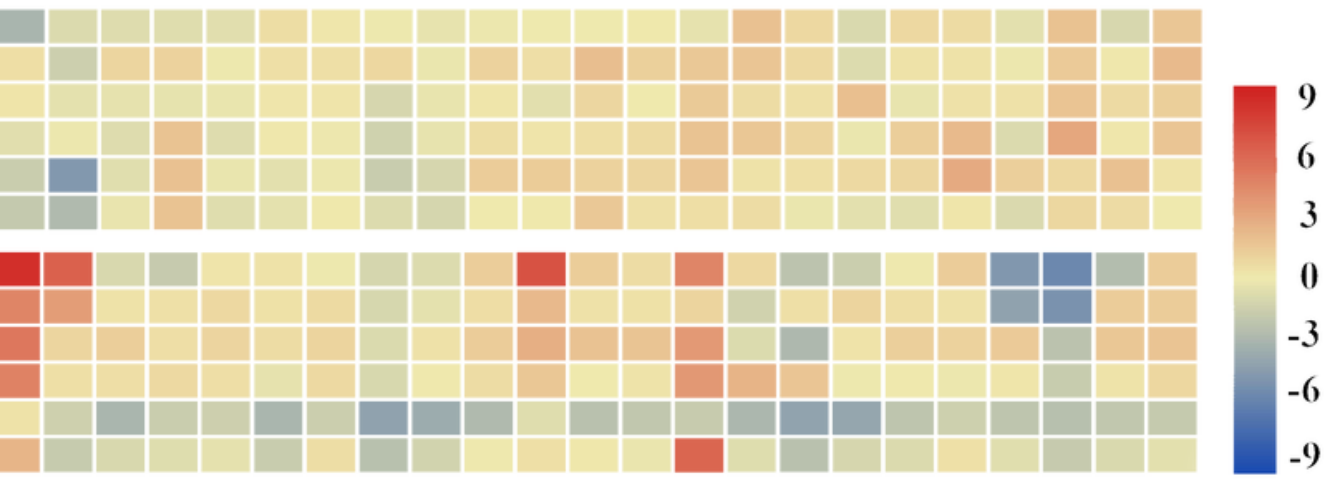

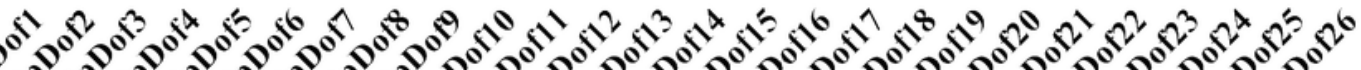

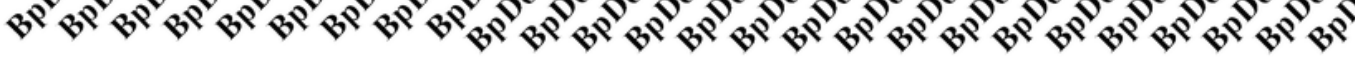




\section{Figure 7}

Analysis of ROS accumulation and cell membrane damage among transgenic and control birch plants.

Birch plants were transiently transformed with 35S: BpDof4, BpDof11, and BpDof17 for overexpression, and empty pROKII was transiently transformed into plants as the control. After treatment with $20 \%$ PEG6000, the overexpression and control lines were individually

stained with NBT to visualize $\mathrm{O}^{2-}$, stained with $\mathrm{DAB}$ to visualize $\mathrm{H}_{2} \mathrm{O}_{2}$ level, and stained with Evans blue to visualize cell membrane damage. 
NBT

WT

pROKII-35S

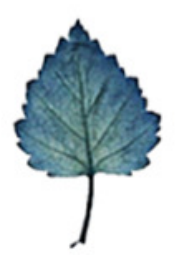

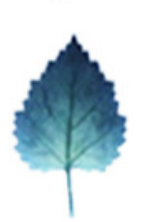

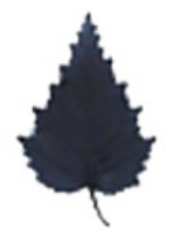

pROKII-BpDof4
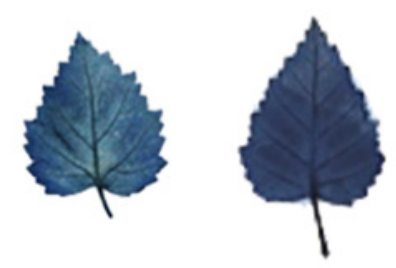

pROKII-BpDof11
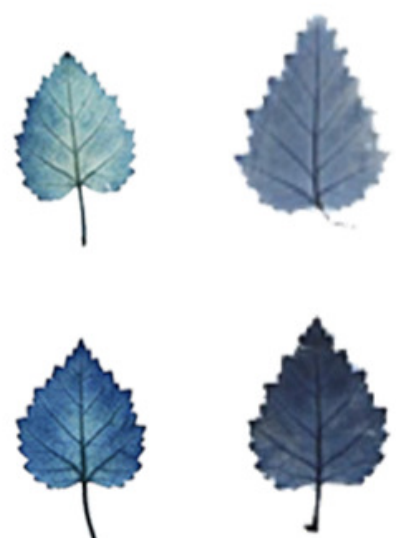

Control

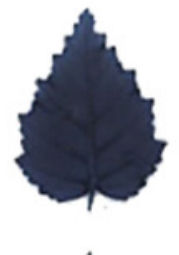

DAB
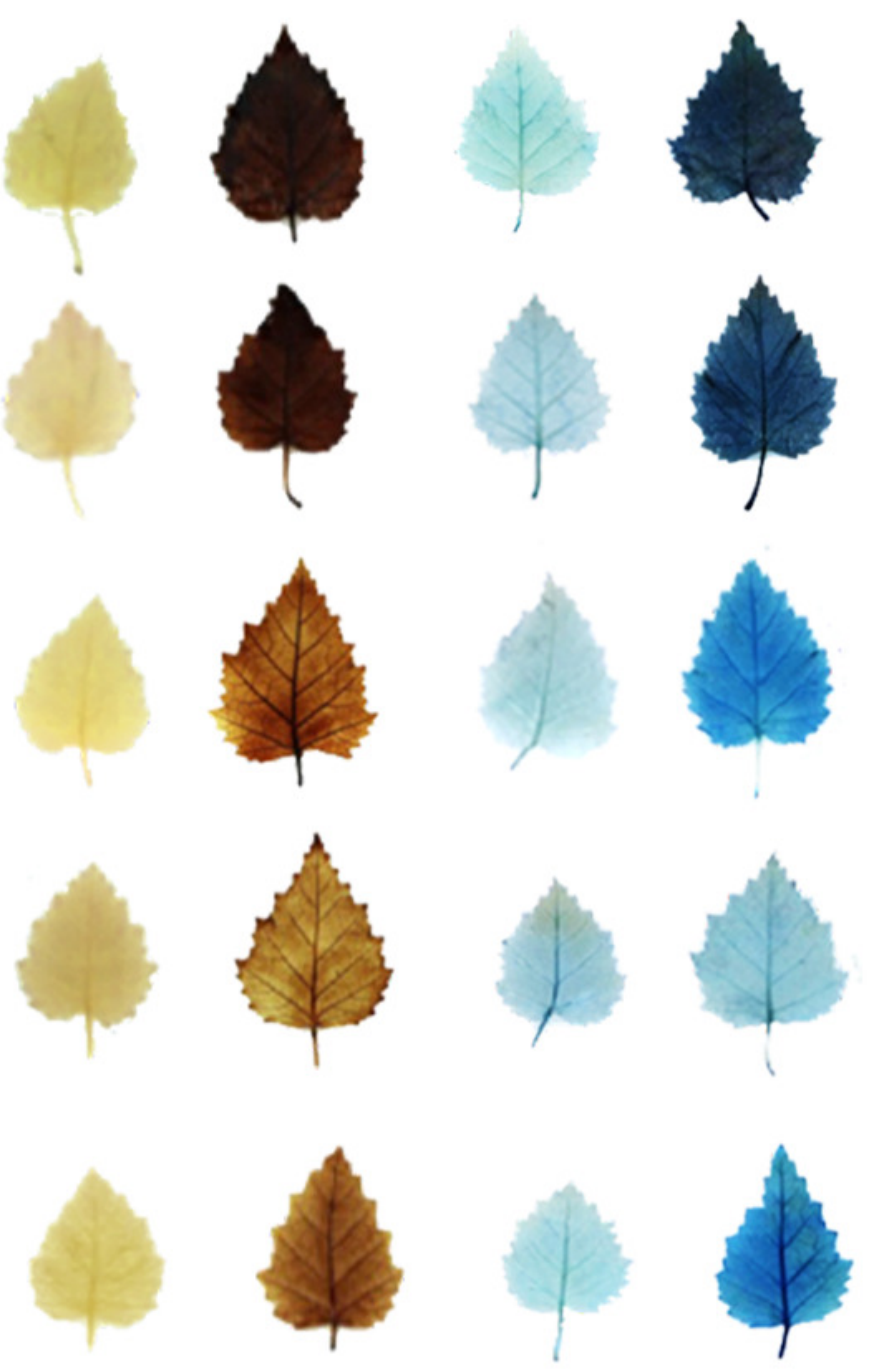

Control PEG

Control PEG 


\section{Figure 8}

Physiological analysis of transgenic BpDof4-, BpDof11-, and BpDof17-overexpressing plants and control plants.

SOD and POD activities, $\mathrm{H}_{2} \mathrm{O}_{2}$ content, and electrolyte leakage in WT and transgenic plants under drought treatment conditions were measured. (A) Measurement of SOD activity. (B) Measurement of POD activity. (C) Measurement of $\mathrm{H}_{2} \mathrm{O}_{2}$ content. (D) Measurement of electrolyte leakage.

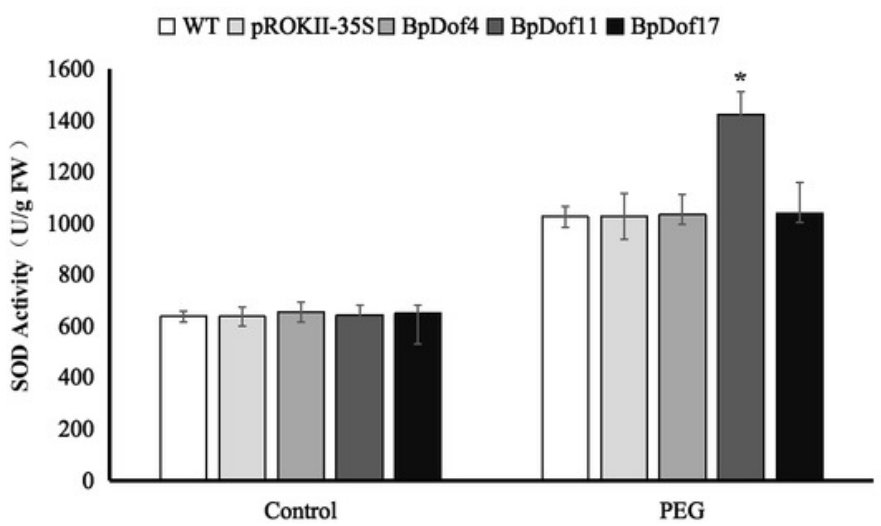

口WT $\square$ pROKII-35S $\square$ BpDof4 $\square$ BpDof11 $\bullet$ BpDof17

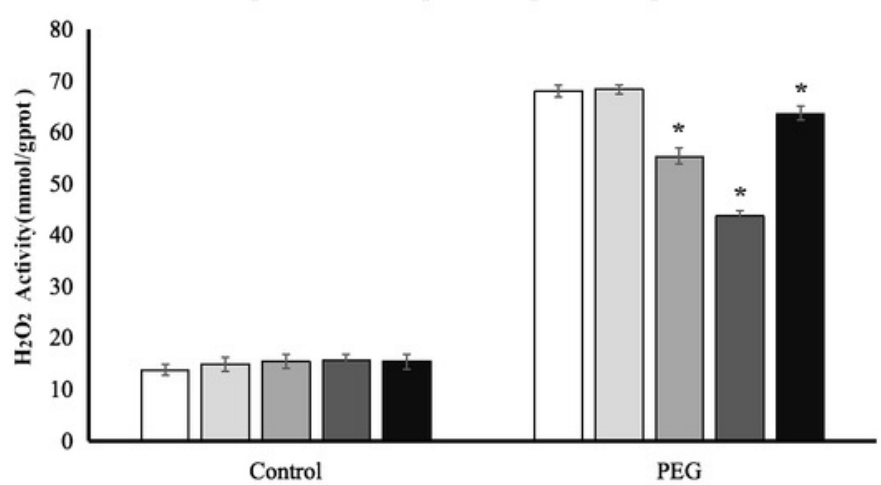

$\square$ WT $\square$ pROKII-35S $\square$ BpDof4 $\square$ BpDof1 1 BpDof17

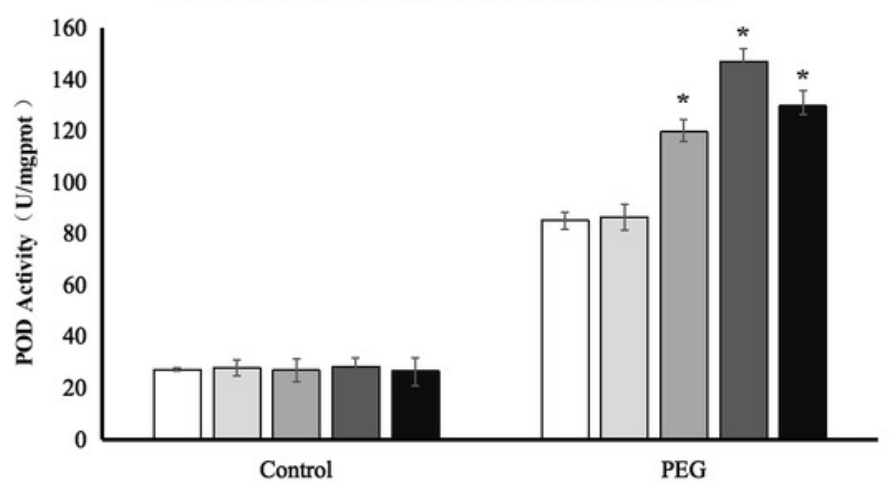

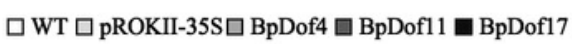

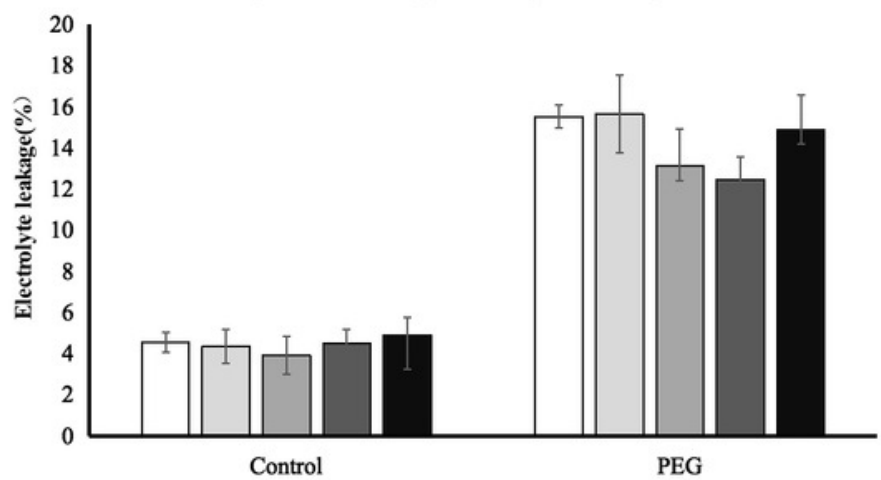

